\title{
The VMC Survey
}

\section{Pulsation periods of dust-enshrouded AGB stars in the Magellanic Clouds ${ }^{\star}, \star \star$}

\author{
M. A. T. Groenewegen ${ }^{1}$, A. Nanni ${ }^{2}$, M.-R. L. Cioni ${ }^{3}$, L. Girardi $^{4}$, R. de Grijs $5,6,7$, V. D. Ivanov ${ }^{8}$, M. Marconi ${ }^{9}$, \\ M.-I. Moretti ${ }^{9}$, J. M. Oliveira ${ }^{10}$, M. G. Petr-Gotzens ${ }^{8,11}$, V. Ripepi ${ }^{9}$, and J. Th. van Loon $^{10}$ \\ 1 Koninklijke Sterrenwacht van België, Ringlaan 3, 1180 Brussels, Belgium \\ e-mail: martin.groenewegen@oma . be \\ 2 Aix Marseille Université, CNRS, CNES, LAM, 38, Rue Frédéric Joliot-Curie, 13388 Marseille Cedex 13, France \\ ${ }^{3}$ Leibniz Institut für Astrophysik Potsdam, An der Sternwarte 16, 14482 Potsdam, Germany \\ 4 Osservatorio Astronomico di Padova-INAF, Vicolo dell'Osservatorio 5, 35122 Padova, Italy \\ 5 Department of Physics and Astronomy, Macquarie University, Balaclava Road, Sydney, NSW 2109, Australia \\ ${ }^{6}$ Research Centre for Astronomy, Astrophysics and Astrophotonics, Macquarie University, Balaclava Road, Sydney, NSW 2109, \\ Australia \\ 7 International Space Science Institute-Beijing, 1 Nanertiao, Zhongguancun, Hai Dian District, Beijing 100190, PR China \\ 8 European Southern Observatory, Karl-Schwarzschild-Str. 2, 85748 Garching bei München, Germany \\ 9 INAF - Osservatorio Astronomico di Capodimonte, Via Moiariello 16, 80131 Naples, Italy \\ ${ }^{10}$ Lennard-Jones Laboratories, School of Chemical and Physical Sciences, Keele University, Keele ST5 5BG, UK \\ 11 Universitäts-Sternwarte, Ludwig-Maximilians-Universität München, Scheinerstr 1, 81679 München, Germany
}

Received 5 December 2019 / Accepted 2 March 2020

\section{ABSTRACT}

Context. Variability is a key property of stars on the asymptotic giant branch (AGB). Their pulsation period is related to the luminosity and mass-loss rate of the star. Long-period variables (LPVs) and Mira variables are the most prominent of all types of variability of evolved stars. The reddest, most obscured AGB stars are too faint in the optical and have eluded large variability surveys.

Aims. We obtained a sample of LPVs by analysing $K$-band light curves (LCs) of a large number of sources in the direction of the Magellanic Clouds with the colours expected for red AGB stars $((J-K)>3$ mag or equivalent in other colour combinations).

Methods. Selection criteria were derived based on colour-colour and colour-magnitude diagrams from the combination of the VISTA Magellanic Cloud (VMC) survey, Spitzer IRAC and AllWISE data. After eliminating LPVs with known periods shorter than 450 days, a sample of 1299 candidate obscured AGB stars was selected. $K$-band LCs were constructed by combining the epoch photometry available in the VMC survey with literature data, were analysed for variability, and fitted with a single period sine curve to derive mean magnitudes, amplitudes, and periods. A subset of 254 stars are either new variables, known variables where the period we find is better determined than the literature value, or variables with periods longer than 1000 days. The spectral energy distributions (SEDs) of these stars were fitted to a large number of templates. For this purpose the SEDs and Spitzer IRS spectra of some non-AGB stars (Be stars, HII regions and young stellar objects - YSOs) were also fitted to have templates of the most likely contaminants in the sample.

Results. A sample of 217 likely LPVs is found. Thirty-four stars have periods longer than 1000 days, although some of them have alternative shorter periods. The longest period of a known Mira in the Magellanic Clouds from Optical Gravitational Lensing Experiment data (with $P=1810 \mathrm{~d}$ ) is derived to have a period of $2075 \mathrm{~d}$ based on its infrared LC. Two stars are found to have longer periods, but both have lower luminosities and smaller pulsation amplitudes than expected for Miras. Mass-loss rates and luminosities are estimated from the template fitting. Period-luminosity relations are presented for carbon (C-) and oxygen (O-) rich Miras that appear to be extensions of relations derived in the literature for shorter periods. The fit for the $\mathrm{C}$ stars is particularly well defined (with 182 objects $)$ and reads $M_{\mathrm{bol}}=(-2.27 \pm 0.20) \cdot \log P+(1.45 \pm 0.54)$ mag with an rms of 0.41 mag. Thirty-four stars show pulsation properties typical of Miras while the SEDs indicate that they are not. Overall, the results of the LC fitting are presented for over 200 stars that are associated with YSOs.

Key words. stars: variables: general - Magellanic Clouds - stars: AGB and post-AGB

\section{Introduction}

At the end of their lives, essentially all low- and intermediate mass stars (roughly $\sim 0.9$ to $\sim 10 M_{\odot}$ on the main sequence) will go through the (super)-asymptotic giant branch (AGB) phase.

^ Full Tables A.1-A.3, and D.1 are only available at the CDS via anonymous ftp to cdsarc.u-strasbg.fr (130.79.128.5) or via http://cdsarc.u-strasbg.fr/viz-bin/cat/J/A+A/636/A48

$\star \star$ Based on observations made with VISTA at ESO under programme ID 179.B-2003.
They end up as $\sim 0.55-1.4 M_{\odot}$ white dwarfs, which means that a large fraction of the initial mass of the star is lost to the interstellar medium. An important characteristic of AGB stars is that they pulsate, and they are classically divided into stars with small amplitudes (the semi-regular variables, SRVs) and the largeamplitude Mira variables. It is now common to use the term long-period variable (LPV) regardless of pulsation amplitude for a pulsating star on the AGB. Pulsation-induced shock waves and radiation pressure on dust is the most promising mechanism to explain wind driving, especially regarding the more 
evolved AGB stars with low effective temperatures, large pulsation amplitudes, and high mass-loss rates (MLRs; see the review by Höfner \& Olofsson 2018).

It was known from observations in the Large Magellanic Cloud (LMC) that Miras follow a period-luminosity (PL) relation (Glass \& Evans 1981; Feast et al. 1989), but the breakthrough came with the advent of micro-lensing surveys. Wood et al. (1999) and Wood (2000) showed that late-type stars in the LMC follow five sequences (labelled A, B, C, D, and E). Subsequent work demonstrated that sequence B consists actually of two sequences ( $\mathrm{B}$ and $\mathrm{C}^{\prime}$ ) and that an additional sequence $\left(\mathrm{A}^{\prime}\right)$, includes possible substructure (Ita et al. 2004; Soszyński et al. 2007). Sequence D consists of long secondary periods (LSPs), while sequence $\mathrm{E}$ is due to binary stars. A theoretical interpretation using linear radial non-adiabatic pulsation models and a population synthesis model showed that sequences $\mathrm{A}^{\prime}, \mathrm{A}, \mathrm{B}+\mathrm{C}^{\prime}$, and $\mathrm{C}$, correspond to third, second, and first overtone and fundamental mode pulsation, respectively (Trabucchi et al. 2017). The LPVs with the largest amplitudes, the classical Miras, dominate sequence $\mathrm{C}$.

The Mira PL-relation has been used as a distance indicator, traditionally inside the Milky Way galaxy in the pre-Gaia era (e.g. Groenewegen et al. 2002; Ramstedt \& Olofsson 2014). The method has gained recent interest in the context of the distance ladder as an independent check on Cepheids or the tip of the red giant branch distances; example are recent works on the mega-maser galaxy NGC 4258 (Huang et al. 2018) or the SN host galaxy NGC 1559 (Huang et al. 2020). Because Miras are as bright as Cepheids in the near-IR (NIR) they will be important secondary calibrators in the upcoming era of groundbased extremely large telescopes and the James Webb Space Telescope.

The currently largest survey of LPVs in the Magellanic Clouds (MCs) is the Optical Gravitational Lensing Experiment OGLE-III catalogue, which contains 1667 Mira stars, 11128 SRVs and 79200 OGLE small-amplitude red giants (OSARGs) in the LMC (Soszyński et al. 2009a), and 352 Mira stars, 2222 SRVs and 16810 OSARGs in the Small Magellanic Cloud (SMC; Soszyński et al. 2011).

However, OGLE and other current surveys in the optical domain will miss the reddest, most obscured AGB stars. At the very end of the AGB the (dust) MLR may become so high that the object becomes very faint, beyond the OGLE I-band detection limit of about $21 \mathrm{mag}$. The dust grains in the circumstellar envelope (CSE) scatter and absorb the emission in the optical to re-emit it in the NIR and mid-infrared (MIR).

These stars are known to exist in the LMC. They were typically selected as having Infrared Astronomical Satellite (IRAS) colours similar to obscured AGB stars in our Galaxy. Wood et al. (1992) reported periods for nine sources in the LMC. Periods range between 930 and $1390 \mathrm{~d}$, with peak-to-peak amplitudes in $K$ between 0.3 and $2.1 \mathrm{mag}$, and $(J-K)$ colours that range from 2.1 to $5.7 \mathrm{mag}$ (an additional 7 LMC and $3 \mathrm{SMC}$ sources were monitored, but showed small amplitudes, and no periods were given). Wood (1998) monitored 12 sources, of which nine were LPVs with periods in the range 530 to $1295 \mathrm{~d}$. Amplitudes in $K$ are between 0.85 and $1.9 \mathrm{mag}$, and colours range from $(J-K)=3.9$ to $6.1 \mathrm{mag}$ (two stars were not detected in $J)$. Similarly, Whitelock et al. (2003) reported periods for two dozen stars with an IRAS identifier. Periods range from 540 to 1390 days, $K$-band amplitudes are between 0.52 and $1.8 \mathrm{mag}$, and $(J-K)$ colours range from 1.9 to 4.0 mag (several are not detected in $J$ ). The faintest stars in these works have (mean light) magnitudes in the range $K=12-13 \mathrm{mag}$.
The ESO public VISTA Magellanic Cloud (VMC) survey is a photometric survey in three filters, $Y, J$, and $K_{s}$ performed at the VISTA telescope using the VIRCAM camera. The latter provides a spatial resolution of $0.34^{\prime \prime}$ per pixel and a non-contiguous field of view of $1.65 \mathrm{deg}^{2}$ sampled by 16 detectors. To homogeneously cover the field of view it is necessary to fill these gaps using a six-point mosaic. This is the unit area of VISTA surveys, called a tile, and it covers an area of $1.5 \mathrm{deg}^{2}$ uniformly with at least two exposures per pixel (Emerson \& Sutherland 2010).

The VMC survey covers an area of approximately $170 \mathrm{deg}^{2}$ (110 tiles) of the MC system and includes stars as faint as 22 mag in $K_{s}(5 \sigma$, Vega mag), see Cioni et al. (2011) for a description of the survey. The large areal coverage and the survey depth are the main strengths of the VMC survey compared to earlier works in the IR. Unlike the VMC papers on classical variables (TypeII and Classical Cepheids, RR Lyrae, e.g. Ripepi et al. 2012, 2015, 2016, 2017; Muraveva et al. 2018), the expected periods for AGB pulsators are much longer than the baseline of the VMC observations. Therefore the VMC data are combined with $K$ band data from the literature when available to increase the time span.

In this paper we inspect $K$-band light curves (LCs) for a large number of candidate dust-obscured AGB stars. The sample is selected based on colour-magnitude and colour-colour criteria derived from the properties of known AGB stars, using both VMC and other IR data.

The paper is organised as follows. Section 2 discusses our selection of the sample of potential LPVs, which is based on different photometric catalogues, and the creation of the $K$-band LCs. Section 3 describes our analysis of the LCs. Section 4 presents the results. The sample of potential LPVs is further reduced by considering the shape of the spectral energy distributions (SEDs).

Preliminary results of this work were shown or used in Groenewegen et al. (2016), Cioni et al. (2017), and Groenewegen \& Sloan (2018, hereafter GS18), but the results on the LC parameters are superseded by this paper.

\section{Sample selection}

In this section the sample of candidate obscured AGB stars is generated. It is based on three different photometric catalogues. The first selection uses only VMC data ${ }^{1}$, but requires a detection in $J$ and $K_{s}$ for reliability (sample 1). The second selection combines the VMC $K_{s}$-band data with WISE data from the AllWISE catalogue (Cutri et al. 2013) (sample 2). The third selection starts with data that are exclusively taken from the Surveying the Agents of a Galaxy's Evolution (SAGE) survey of the MCs, and then searches for a possible counterpart in the VMC data (sample 3). During this selection procedure, only the mean magnitudes as given by the VISTA Science Archive (VSA; Cross et al. 2012) are used, and the quality flags indicating warnings and possible problems (the ppErrBits) are not considered. Only in the second step are the time-resolved VMC $K_{s}$-band data used and combined with literature data to produce an LC.

\subsection{Sample 1: VMC data alone}

Figure 1 shows the VMC $\left(K_{s},\left(J-K_{s}\right)\right)$ colour-magnitude diagram (CMD) for a region in the Bridge between the two Clouds and a region located in the bar of the LMC. The region in the

All VMC data refer to observations obtained until the end of September 2016, including complete observations of the SMC. 

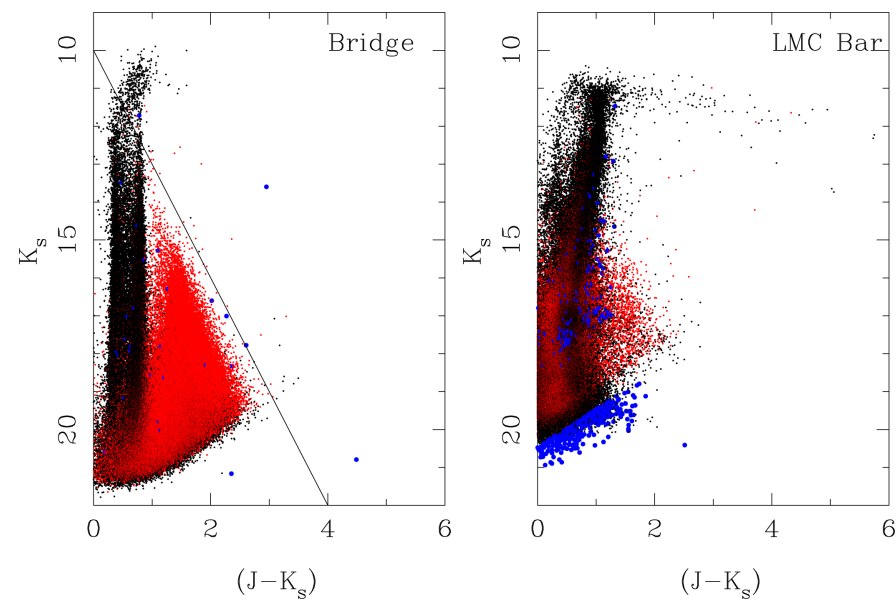

Fig. 1. VMC $\left(K_{s},\left(J-K_{s}\right)\right)$ CMD in a region in the Magellanic Bridge (left-hand panel; a strip at $42^{\circ}<\mathrm{RA}<52^{\circ}$ and $-72.5^{\circ}<$ Dec $<-73.8^{\circ}$ ) and in the LMC bar (right-hand panel; a box defined as $80^{\circ}<\mathrm{RA}<82^{\circ}$, and $-69^{\circ}<$ Dec $<-70^{\circ}$ ). Objects that are (probable) stars are indicated in black, and (probable) galaxies (extended objects) are indicated in red. Objects classified differently are indicated in blue. The line, $K=3 \cdot(J-$ $K)+10 \mathrm{mag}$, indicates the approximate boundary of galaxies.

Bridge is used to delineate the locus in colour space occupied by extended objects, most of which are background galaxies, but can also result from blends (colour-coded in red) $)^{2}$, while the region in the bar shows the well-known sequence of AGB stars redder than $\left(J-K_{S}\right) \sim 1.5 \mathrm{mag}$ at relatively bright magnitudes. At the brightest level $\left(K_{s} \lesssim 10.5 \mathrm{mag}\right)$, we note the effect of saturation. This is not expected to influence the selection of obscured AGB stars for which the distribution in the CMD bends to faint magnitudes. This is shown more clearly in Fig. 2.

In a subsequent step, all (about 7000) stars in VMC with a detection in $J$ and $K_{s},\left(J-K_{s}\right)>1.5 \mathrm{mag}$, and $K_{s}<3 \cdot(J-$ $\left.K_{s}\right)+10$ mag were selected to represent candidate obscured AGB stars. Their CMD distribution is shown in the left-hand panel of Fig. 2, and it shows that there is still a high contribution by background galaxies and other extended objects. This CMD also clearly exhibits the sequence of increasing mass loss along the AGB. A fit was made to this sequence, $K_{s}=10.318+0.287$. $\left(J-K_{S}\right)+0.0306 \cdot\left(J-K_{S}\right)^{2} \mathrm{mag}$, and this relation, which was shifted by two magnitudes to fainter magnitudes to delineate the approximate lower boundary of this sequence, is shown as well.

Another consideration in selecting the stars is that the primary interest is in studying the reddest pulsating AGB stars. These objects that are too faint for current optical surveys such as OGLE-III and OGLE-IV, which have a limiting magnitude in the $I$-band around 20-21 mag.

GS18 fitted the SEDs and spectra taken with the Spitzer Infrared Spectrograph (IRS, Houck et al. 2004) of almost 400 AGB and red supergiant (RSG) stars in the MCs, and determined periods for a large fraction of them. The periods were derived by analysing existing optical data (from the OGLE, Massive Compact Halo Objects (MACHO), and Expérience de Recherche d'Objets Sombres (EROS) surveys), or data in the $K$ and $K_{s}$ bands (including VMC data) and in the WISE $W 2$ band for the reddest objects.

The right-hand panel of Fig. 2 shows the $I,\left(J-K_{s}\right)$ CMD for about $150 \mathrm{O}$ - and C-rich AGB stars in the MCs with a pulsa-

\footnotetext{
2 Indicated by the mergedClass parameter which is -9 for a saturated source, -3 for a probable galaxy, -2 for a probable star, -1 for a star, 0 for noise, and +1 for a galaxy.
}
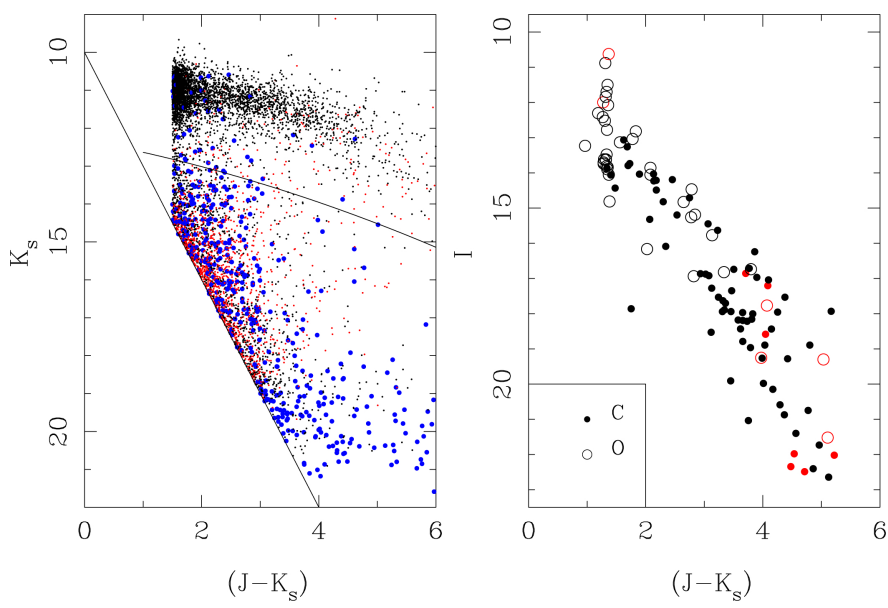

Fig. 2. Left-hand panel: all VMC stars with a detection in $J$ and $K_{s}$, redder than $\left(J-K_{s}\right)=1.5 \mathrm{mag}$ and above the line $K_{s}=3 \cdot\left(J-K_{s}\right)+10 \mathrm{mag}$. The second-order polynomial is a fit to the red sequence of AGB stars, shifted downward by two magnitudes for comparison purposes (see text). Objects that are (probable) stars are indicated in black, and (probable) galaxies are indicated in red. Objects classified differently are indicated in blue. Right-hand panel: synthetic photometry from GS18 for 29 SMC and 133 LMC O- (open circles) and C-rich (filled circles) AGB stars with pulsation periods longer than 450 days. The pulsation periods of the objects marked in red are derived from $K / K_{s}$ or WISE-band data, and the black points are derived from optical data (OGLE, EROS, and $\mathrm{MACHO}$.

tion period longer than 450 days, based on synthetic photometry, that is, the simulated magnitudes taking into account the filter transmission curves and photometric zero-points in many filters using the best-fitting model to the SEDs in GS18. Marked in red are stars where the pulsation period is based on $K / K_{S}$ or $W 2$ data, the other periods are derived from optical data (mainly OGLE). The diagram shows that the $I$-band limit of $20 \mathrm{mag}$ is reached for stars that have a $\left(J-K_{s}\right)$ colour of $\sim 4$ mag.

To have sufficient overlap with the optical surveys and to be on the conservative side, a limit of $\left(J-K_{S}\right)=3$ mag was chosen. Sample 1 consists of the 857 stars in the VMC survey that have a detection in $J$ and $K_{s},\left(J-K_{s}\right)>3$ mag and $K_{s}<12.318+$ $0.287 \cdot\left(J-K_{s}\right)+0.0306 \cdot\left(J-K_{s}\right)^{2} \mathrm{mag}$.

\subsection{Sample 2: VMC and WISE data combined}

While sample 1 has the advantage that it uses exclusively data from the VMC survey, the reddest AGB stars by their very nature will become $J$-band dropouts for high enough MLRs. The aim of constructing samples 2 and 3 was to combine VMC $K_{s}$-band data with data at longer wavelengths, in particular, WISE (sample 2) or IRAC (sample 3). The advantage of WISE is that it is an allsky survey, covering the complete area observed by the VMC survey.

The AllWISE catalogue (Cutri et al. 2013) is available as a table in the VSA and the queries were therefore performed within the VSA. A search radius of $1^{\prime \prime}$ was used and an error on the $W 1$ magnitude of less than 0.2 mag was imposed (equivalent to $w 1$ snr $>5$ ).

The left-hand panel of Fig. 3 shows the $\left(W 1, K_{s}-W 1\right)$ CMD for a region in the Bridge, with the same colour-coding for background galaxies and stars as in Fig. 1. The line $W 1=$ $1.0 \cdot\left(K_{s}-W 1\right)+10.5$ indicates the approximate boundary beyond which (at bright magnitudes and red colours) the number of galaxies significantly decreases. In the second panel, all sources 

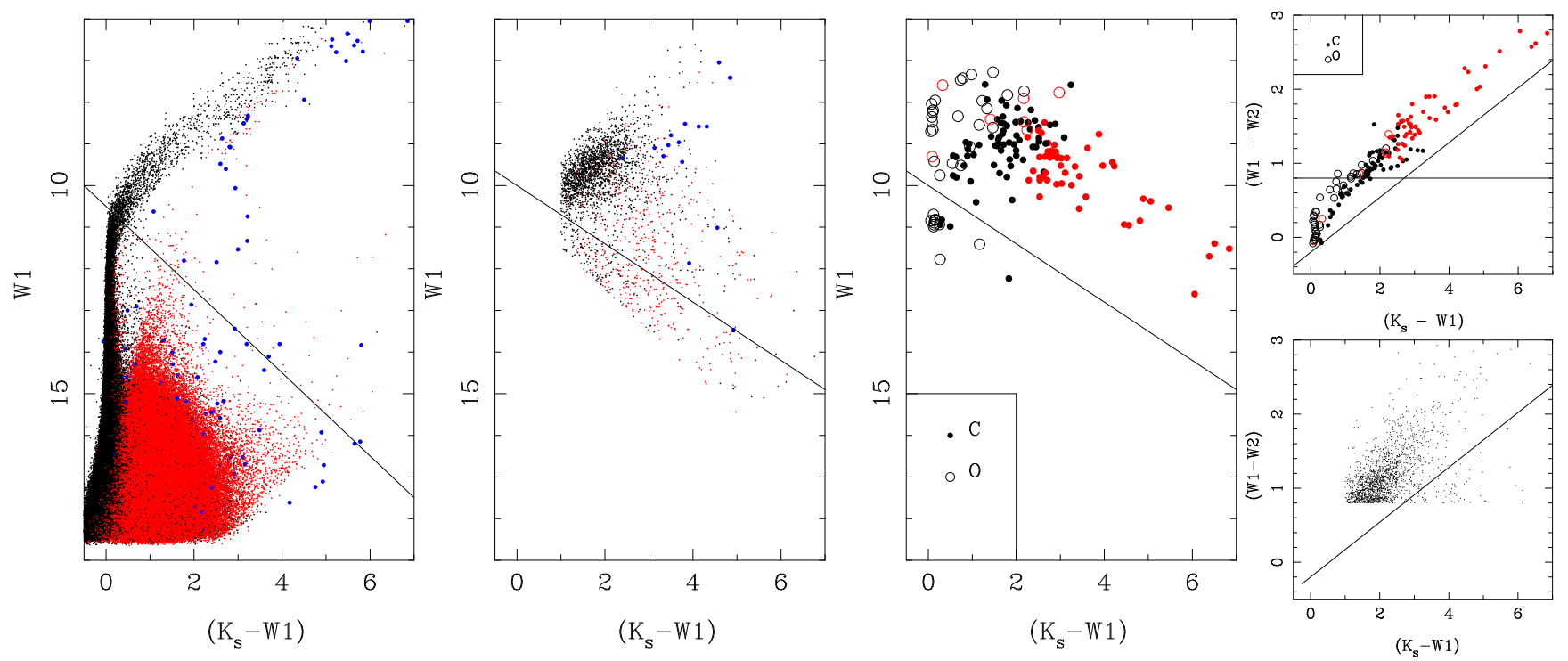

Fig. 3. WISE-VMC $\left(W 1, K_{s}-W 1\right)$ CMD and $(W 1-W 2)-\left(K_{s}-W 1\right)$ CCDs. Left-most panel: CMD in a region between the SMC and LMC (a strip at $30^{\circ}<\mathrm{RA}<60^{\circ}$ ). The line $W 1=1.0 \cdot(K-W 1)+10.5$ mag indicates the upper boundary of the region predominantly occupied by galaxies. Second panel from the left: all WISE-VMC sources that are brighter than the boundary line in the left-hand panel, $\left(K_{s}-W 1\right)>1 \mathrm{mag}$ and $(W 1-W 2)>0.5$ mag. Objects that are (probable) stars are indicated in black, and (probable) galaxies (extended objects) are indicated in red, while objects classified differently are indicated in blue in these two panels. The provenance of the line in the second panel is explained in the next panel. The corresponding CCD is shown in the bottom half of the right-most panel. Third panel from the left: synthetic CMD based on GS18 for 29 SMC and 133 LMC O- (open circles) and C-rich (filled circles) AGB stars with pulsation periods longer than 450 days. The pulsation periods of the objects marked in red are derived from $K / K_{s}$ or WISE-band data, and the black points are derived from optical data (OGLE, EROS, and MACHO). The line $W 1=0.7 \cdot\left(K_{s}-W 1\right)+10.0 \mathrm{mag}$ is the empirical lower boundary to the reddest AGB stars, and it is also shown in the second panel from the left. The corresponding CCD diagram is shown in the top half of the right-most panel with the lines $(W 1-W 2)=0.8$ mag and $(W 1-W 2)=0.37 \cdot\left(K_{s}-W 1\right)-0.2 \mathrm{mag}$, which indicates the empirical lower boundary to the reddest AGB stars, and is also shown in the panel below it.

are shown which have $\left(K_{s}-W 1\right)>1 \mathrm{mag},(W 1-W 2)>0.5 \mathrm{mag}$, and are brighter than this boundary. The corresponding colourcolour diagram (CCD) is shown in the bottom half of the rightmost panel.

The third panel from the left shows a synthetic CMD based on the AGB stars studied by GS18. The line $W 1=0.7$. $\left(K_{s}-W 1\right)+10.0$ is the empirical conservative lower boundary to the reddest AGB stars, and is also shown in the second panel from the left. The corresponding CCD is shown in the top-half of the right-most panel with the lines $(W 1-W 2)=0.8$ mag and $(W 1-W 2)=0.37 \cdot\left(K_{s}-W 1\right)-0.2 \mathrm{mag}$.

The final selection is based on a detection in $K_{s}$, a signalto-noise ratio $(\mathrm{S} / \mathrm{N})$ in the $W 1$ band $>5,(W 1-W 2)>0.8 \mathrm{mag}$, $W 1<0.7 \cdot\left(K_{s}-W 1\right)+10.0 \mathrm{mag}$, and $(W 1-W 2)>0.37$. $\left(K_{s}-W 1\right)-0.2 \mathrm{mag}$ for a total of 1317 sources.

\subsection{Sample 3: SAGE data first}

SAGE (Meixner et al. 2006) and SAGE-SMC (Gordon et al. 2011) are surveys of the LMC and SMC with the Spitzer telescope. The advantage of Spitzer is that it has a smaller point spread function than WISE, and confusion is less of a problem. On the other hand, the SAGE observations do not entirely cover the area sampled by the VMC survey. The combined LMC and SMC source catalogue in the [3.6], [4.5], [5.8], and [8.0] bands is available electronically ${ }^{3}$.

Figure 4 shows in the left panel the ([8.0], [3.6]-[8.0]) CMD of all sources with detections in all four bands. The region occu-

\footnotetext{
ViZieR catalogue II/305/archive.
}

pied by background galaxies is clearly visible, as is a tail of bright red AGB stars.

Boyer et al. (2011) used this diagram to select very red AGB stars, and we followed their work in selecting stars with [3.6]-[8.0] $>0.8 \mathrm{mag}$. Our elimination of background galaxies is less conservative than theirs, and we selected objects above the line [8.0] $<13.0-([3.6]-[8.0])$ (for [3.6]-[8.0] $<4 \mathrm{mag}$ ), and $[8.0]<9.0+([3.6]-[8.0]-4.0)$ mag for redder objects.

The ([3.6]-[4.5], [5.8]-[8.0]) CCD of sources that meet these conditions is plotted at the top of the right-hand panel of Fig. 4. The bottom panel shows the synthetic photometry based on GS18; a region delineated by the lines [3.6]-[4.5] $>-0.6$. $([5.8]-[8.0])+0.6($ for $([5.8]-[8.0])<1 \mathrm{mag})$, and [3.6] $-[4.5]>$ $1.7 \cdot([5.8]-[8.0])-1.7$ (for $([5.8]-[8.0])>1 \mathrm{mag})$ is defined. The initial sample consisted of 1958 SAGE sources that meet these constraints.

The next step was the correlation with the VMC database. After inspecting the results using a search radius of $1^{\prime \prime}$, we determined the final correlation by returning all VMC counterparts within $0.8^{\prime \prime}$ of the SAGE coordinates. Multiple counterparts were listed for ten sources, but there was no reason not to take the closest match in any case. In the sample 35 SAGE stars had no counterpart in VMC, and in eight cases the counterpart lacked $K_{s}$-band data, leaving 1915 objects.

The selection so far was based on SAGE colours and positions. An additional consistency check between the IRAC and the $K_{s}$-band VMC magnitude was performed. Based on the synthetic photometry from GS18, we imposed a limit of $K_{s}-$ [3.6] > $1.5 \mathrm{mag}$ (which is consistent with the limit $\left(J-K_{s}\right)>3 \mathrm{mag}$ in selecting sample 1). This removed over 300 sources for a final sample size of 1562 . 

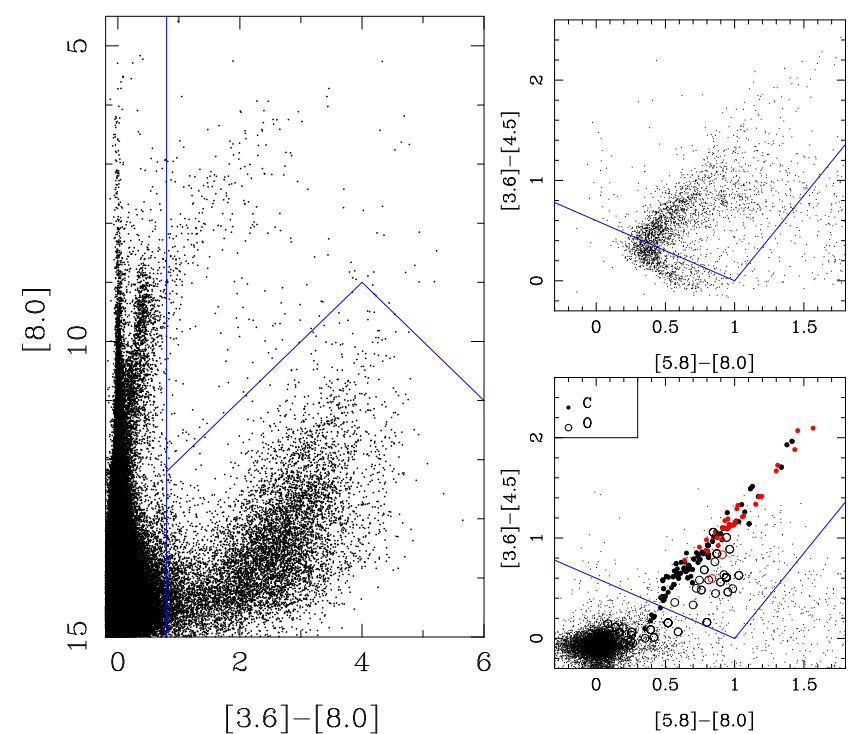

$[5.8]-[8.0]$

Fig. 4. Left-hand panel: ([8.0], [3.6]-[8.0]) CMD of LMC and SMC objects in the SAGE survey. Only every tenth star is plotted. The line at $[3.6]-[8.0]=0.8 \mathrm{mag}$ indicates the blue limit of red AGB stars, and the two other lines indicate the limits of the region that is mainly occupied by background galaxies (see text). Top right-hand panel: ([3.6]-[4.5], [5.8]-[8.0]) CCD of all sources that are located in the upper right of the CMD delineated by these lines. Bottom right-hand panel: same diagram with synthetic CCD based on GS18 for 29 SMC and 133 LMC O(open circles) and C-rich (filled circles) AGB stars with pulsation periods longer than 450 days. The pulsation periods of the objects marked in red are derived from $K / K_{s}$ or WISE-band data, and the black points are derived from optical data (OGLE, EROS, and MACHO). The background sources in this panel are randomly picked from the $\mathrm{S}^{3} \mathrm{MC}$ survey (Bolatto et al. 2007).

\subsection{Final sample}

\subsubsection{Sample statistics}

The three samples were combined and together contain 2014 unique sources. This sample is almost of the same size as that of the known Mira population in the MCs, indicating that the initial sample selection based on colour criteria was not very restrictive.

As the main interest in the present paper is determining new red and (very) long-period pulsating stars, the next step was a correlation with lists of known LPVs. To be able to compare the properties (in terms of period, amplitude, etc.) of known Miras and LPVs with the new objects, known LPVs with periods longer than 450 days were kept, while LPVs with shorter periods were removed. This is an arbitrary choice but a good compromise, because including stars with shorter periods would increase the sample by including stars that would essentially never become optically obscured and where the period from the relatively poorly sampled IR LC would never be able to compete in quality with period(s) determined from OGLE data. At the same time the cut at 450 days allows overlap with OGLE to verify our procedures and compare periods and amplitudes.

A comparison with the OGLE-III catalogue of LPVs in the LMC (Soszyński et al. 2009a) and SMC (Soszyński et al. 2011) removed about 450 stars and kept about 480 LPVs with periods longer than 450 days. Similar correlations were made with the list of MACHO-detected LPVs in the LMC (Fraser et al. 2008) and the catalogues of LPVs detected in the EROS-2 survey (Spano et al. 2011; Kim et al. 2014).

\subsubsection{Light curves}

The final sample of stars for which the LCs were analysed contains 1299 objects, and for these, the $K_{s}$-band epoch data were retrieved from the VSA archive. The information for all these objects is given in Appendix A. Table A.1 lists general information on the objects: the right ascension and declination (from VMC, all epochs in this paper are J2000), some common names and object type (from SIMBAD ${ }^{4}$ ), (selected) spectral types (from Skiff 2014), the variability type as listed in the second Gaia data release (GDR2, Gaia Collaboration 2018; Holl et al. 2018), and the classification based on the MIR Spitzer IRS spectrum; see the footnote to the table for more information. Table A.2 lists known periods and related information from the literature, while Table A.3 lists the results of the period analysis (see Sect. 3 for details).

The LCs consist of the VMC $K_{s}$-band data with $K / K_{s}$-band data found in the literature added (as detailed in Cols. 7 and 8 of Table A.3). For some of the brightest stars the VMC photometry is influenced by non-linearity or saturation. A correction is applied in the VISTA pipeline for this (Irwin 2009), but for the brightest stars, it does not correct sufficiently. In the LC fitting this was accounted for by shifting the VMC $K_{s}$-band photometry to externally available photometry (see the remarks in Col. 10 of Table A.3). The shift was determined from the difference in the median of the VMC and the external photometry. The shifted VMC photometry was also assigned an error bar of $0.25 \mathrm{mag}$.

No attempt was made to bring the various $K$-band measurements into a common system. The main reason is that colour transformations from one system to another almost always depend on the $(J-K)$ colour (Carpenter 2001; Koen et al. 2007). A $J$-band magnitude observed simultaneously with the $K$-band data is not always available, the error on the $J$-band magnitude is typically large for these red sources, and the $(J-K)$ colour is outside the range considered in the transformation formula. This would make a colour transformation particularly complicated and uncertain in many cases. This is a limitation, and in Appendix B we estimate the possible effect and conclude that this is typically smaller than the error bar quoted for mean magnitudes, amplitudes and periods in Table A.3. In the following text we refer to the $K$ band for simplicity unless we address a specific survey.

\section{Modelling}

The automatic analysis of the LCs was made with the Fortran codes available in numerical recipes (Press et al. 1992) as described in Appendix A of Groenewegen (2004). The codes were tuned at that time to analyse OGLE-II LCs with hundreds of data points, while the current LCs have between 6 and 73 data points $(92 \%$ of the stars in the sample have 15 or more data points). This complicates determining the pulsation period through Fourier analysis (using the subroutine FASPER). Therefore the LCs of most stars in the sample were analysed manually with the code PERIOD04 (Lenz \& Breger 2005) as well. After an initial guess of the period was determined (either through the automatic routine, a period found in the literature, or from the manual fitting of the LC) a function of the form

$$
K(t)=K_{0}+A \sin \left(2 \pi t e^{f}\right)+B \cos \left(2 \pi t e^{f}\right)
$$

was fitted to the LC using the weighted linear least-squares fitting routine MRQMIN. This results in the parameters listed in

\footnotetext{
4 As of January 2019.
} 

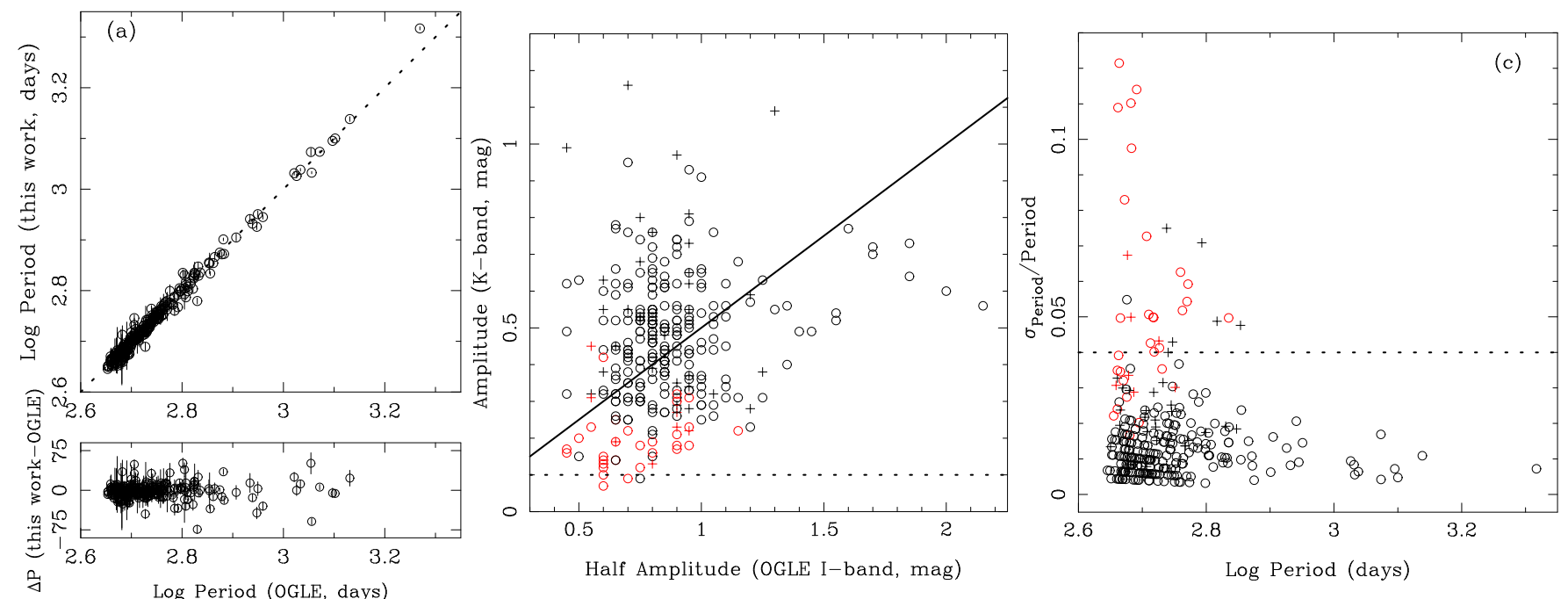

Fig. 5. Pulsation properties of 294 confirmed Miras by OGLE compared with our results. Left panel a: directly compares the periods in the upper part; the dotted line marks the one-to-one relation. The lower part indicates the difference in periods as a function of the period found by OGLE. The star at $P=1858 \mathrm{~d}(\log P=3.26)$ in the top panel is located outside the plot at $\Delta$ Period $=216 \mathrm{~d}$ in the bottom panel. Middle panel $b$ : compares the amplitude in the $K$ band to that in the $I$ band. The OGLE team defines amplitude as the minimum-to-maximum variation, while we use the traditional definition (see Eq. (1)). Stars with a $\chi_{\mathrm{r}}^{2}>430$ are marked by a plus sign, and stars where the error on the $K$-band amplitude is larger than the amplitude are marked in red. The dotted line indicates the cut-off value of $0.1 \mathrm{mag}$ we used to select the maximum number of known Miras from our sample. The solid line has a slope 0.5 to guide the eye. Right-hand panel $c$ : relative error of the period determination against the period. Stars with a $\chi_{\mathrm{r}}^{2}>430$ are marked by a plus sign, and stars where the error on the $K$-band amplitude is larger than the amplitude are marked in red. The dotted line indicates the cut-off value of $4 \%$ error on the period determination we used to select Miras from our sample.

Table A.3, mean magnitudes, periods (exp $(-f))$, and amplitudes $\left(\sqrt{A^{2}+B^{2}}\right)$ with their associated uncertainties. Equation (1) implies that the LC can be described by a single period. It is well known that the LCs of LPVs are not strictly single-periodic. The OGLE team typically fits three periods to the LC. However with the limited number of data points available in the IR LCs we are not able in general to comment on the presence of more than one period. The exception is the few stars that have been monitored extensively from the ground. Table A.3 sometimes lists a long secondary period (LSP). Those were derived from the manual fitting of the LC using PERIOD04.

When no initial period was known from the literature, it was taken from the Fourier analysis. The reliability of this period determination (also) depends on the number of data points in the LC. We selected from Table A.3 stars without an initial period listed and a $S / N>3$ on the final period and amplitude. This shows that the minimum number of useable data points is 12 .

A comparison of the LC with the fit sometimes suggested that alternative periods may be possible as well. These cases were inspected by manual fitting of the LC using PERIOD04, and alternative periods are sometimes indicated in Col. 9 of Table A.3.

The table also includes the reduced $\chi^{2}$, defined as $\chi_{\mathrm{r}}^{2}=$ $\sum_{\mathrm{i}}\left(\left(\left(m_{\mathrm{i}}-o_{\mathrm{i}}\right) / \sigma_{\mathrm{o}_{\mathrm{i}}}\right)^{2}\right) /\left(N-N_{\mathrm{p}}\right)$, with $m, o$, and $\sigma_{\mathrm{o}}$ indicating the model magnitude, the observed magnitude, and the error, $N$ is the number of data points, and $N_{\mathrm{p}}=1$ or 4 , depending on whether Eq. (1) is fitted without or with the period. The $\chi_{\mathrm{r}}^{2}$ in Table A. 3 ranges from 0.2 to 3900 , with a median of 70 . These values are far higher than an expected value of unity for a model that described the data correctly and with correct estimates of the error bars. This probably indicates that the internal error bars of the $K_{s}$ band photometry in the VSA (typically at the millimagnitude level) are likely underestimated when compared to other photometry on an absolute external scale, but also that differences in filter curves between the different sets of photometry, and the simplicity of the model (a single period) play a role. In the next section we perform a selection that eliminates stars with a $\chi_{\mathrm{r}}^{2}>430$, which represents about $10 \%$ of the sample.

\section{Analysis and selection of new red LPVs}

\subsection{Comparison to known Miras}

The next step was a comparison of the results of the IR LC fitting to the periods of known Miras from OGLE-III. To compare between the OGLE and the IR pulsation properties we selected from Table A.2 the stars classified by OGLE to be Miras whose amplitude for the second listed period is smaller than that listed for the primary period (as given by OGLE). As mentioned above, many LPVs show an LSP, sometimes with a large amplitude, and this choice enables a more direct comparison of periods and amplitudes. After removing three stars for which our analysis revealed no significant period, 294 stars remain to make the comparison, and the results are shown in Fig. 5. The panel on the left compares the primary period found by OGLE to the period we derived. The dotted line marks the one-to-one relation and shows that the agreement is very good in most cases. The middle panel compares the amplitudes in the $K$ band as found in our analysis with the amplitude in the $I$ band as found by OGLE. There is no strong correlation. The cut-off in $I$-band amplitude reflects the common definition (as used also by the OGLE team) that the $I$-band amplitude of Miras is expected to be larger than $0.9 \mathrm{mag}$. The solid line is plotted to guide the eye and indicates an amplitude in $K$ that is half that in $I$. The right-hand panel shows the relative error in the period determination plotted against period. The period determination of most stars is better than a few percent. The middle and right-hand panels identify outliers by the red symbols, stars where the amplitude is ill defined (the error on the amplitude is larger than the amplitude itself), and by plus signs stars for which the $\chi_{\mathrm{r}}^{2}$ in the LC fitting is $>430$. Using the following selection criteria, we selected 291 of the 294 known 
Miras (based on I-band data) with low amplitude secondary periods as those for which the error in the period determination is better than $4 \%$, the error in the amplitude smaller is than the amplitude itself, the amplitude is larger than $0.1 \mathrm{mag}$ in the $K$ band, and the $\chi_{\mathrm{r}}^{2}$ in the LC fitting procedure is smaller than 430.

When these criteria were applied to the full sample (we also enforced $P>100$ days to eliminate a few known Type-II Cepheids), 634 objects of the 1299 showed pulsation properties as derived from the IR LCs that were consistent with those of known Miras (as defined based on I-band data). This includes the 291 stars that were used to define the criteria, but also known OGLE LPVs that were not used in the comparison (those with larger LSP amplitudes). In addition, for some objects, the classification of the Spitzer IRS spectrum indicates that they are not AGB stars (see Table A.1). The amplitude cut-off of $0.1 \mathrm{mag}$ does not imply that these are Miras (as opposed to SRVs). They are (candidate) LPVs, and we did not distinguish between the two classes. The middle panel in Fig. 5 shows considerable scatter between the amplitude in the $I$ and $K$ band, but it also demonstrates that the majority of stars that have been classified as Miras based on $I$-band data have $K$-band amplitudes $>0.2 \mathrm{mag}$, in line with expectations.

In a subsequent step, all known OGLE objects were removed, with the exception of stars with periods longer than 1000 days because they are of prime interest here. This limit for a long period is arbitrary, but a limit of 1000 days has also recently been used by Menzies et al. (2019). Stars detected by MACHO or EROS (and not by OGLE) were also removed when the period listed by these surveys was in good agreement with the period found here (and not longer than 1000 days). Although these sources were not retained for further detailed analysis, the pulsation properties derived from the LC fitting are still available in Table A.3.

In a final step, the Spitzer IRS spectra of all stars classified in the literature as not being of an O- or C-rich AGB star (see Table A.1) were inspected visually using the CASSIS tool ${ }^{5}$ (combined atlas of sources with Spitzer IRS spectra, Lebouteiller et al. 2011). All spectra appeared consistent with the literature classification, and thus unlike those of $\mathrm{O}$ - or $\mathrm{C}$ rich AGB stars. Therefore they were removed from the sample of candidate LPVs. The exception is SMP LMC 11 (72.907608 $-67.088019)$. Although it is classified as a planetary nebula (PN) in SIMBAD, and the literature classification of its Spitzer IRS spectrum is that of a C-rich post-AGB star, its spectrum is technically speaking consistent with that of a very red C-rich AGB star. It was therefore retained. At this point, the sample of stars that was investigated further was reduced to 254 .

\subsection{Creating and fitting the SEDs}

As a further step in distinguishing AGB stars from non-AGB stars, the SEDs of the 254 selected stars were constructed and fitted to a large sample of synthetic template SEDs of AGB stars and some non-AGB stars (created from some of the non-AGB stars previously discarded that have MIR spectra).

The procedure is largely outlined in GS18. Photometry was collected from AllWISE, SAGE, Akari, IRAS, OGLE, and GDR2. In the NIR we used the mean $K$-band magnitude derived from the LC fitting. The VMC $Y$ and $J$ magnitude were downloaded from the VSA. The $K_{s}$ band listed in the VSA was also downloaded and assigned a large uncertainty of $0.1 \mathrm{mag}$ so as to give this point less weight in the template fitting. As mentioned

\footnotetext{
https://cassis.sirtf.com/
}
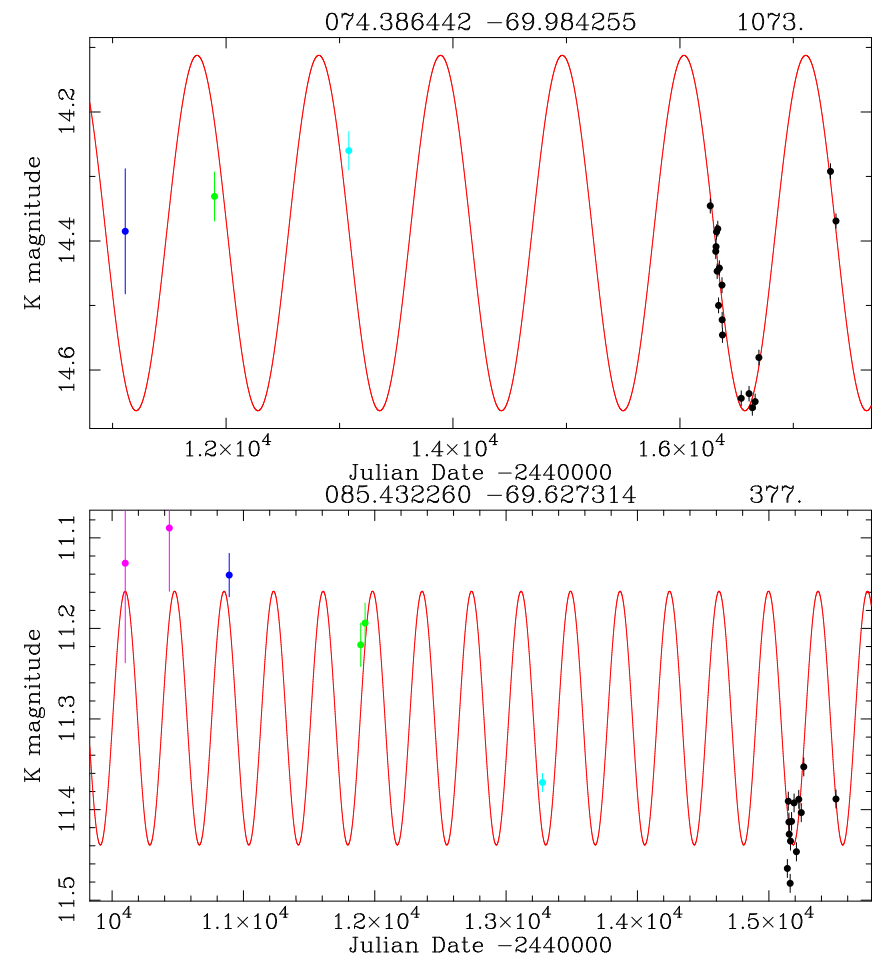

Fig. 6. Examples of LCs of objects where the literature classification and the template fitting indicate a non-AGB nature. Top panel: probable YSO object 2MASS J04573274-6959032 (based on a photometric classification; Table 10 in Gruendl \& Chu 2009), and bottom panel: B1 emission-line star [BE74] 621. Data points (with error bars) in black show the VMC, green points represent $2 \mathrm{MASS}$, dark blue points 2MASS-6X, and light blue points the IRSF.

in Sect. 2.4.2, for some of the brightest stars the VMC photometry is influenced by non-linearity or saturation. For the SEDs the corresponding data points are plotted with large error bars (arbitrarily set at $0.25 \mathrm{mag}$ ) to identify them immediately and in order to avoid affecting the fitting procedure. In some cases the $Y$ and $J$ magnitudes may also be affected by saturation, and these were also assigned large error bars.

The magnitudes were then fitted to the synthetic photometry of several hundred templates. This indicates with high reliability whether the SED of a star is consistent with that of an O-rich, C-rich, or young stellar object (YSO) or other non-AGB objects. Details are given in Appendix C. The SEDs of all 254 objects with their best-matching template are plotted in Fig. C.2.

Based on all available information (spectral type, MIR spectral classification, and template fitting), the 254 stars were divided into 217 most likely AGB stars, and 37 most likely non-AGB stars; see Table C.2. The latter group is no longer discussed, although it contains sources with variability properties that have similarities to those of LPVs. Figure 6 shows the LCs of a YSO and a B1 emission-line star as examples. Table A.1 lists a total of about 200 sources that have been associated with YSOs. An analysis of the VMC-based IR LCs of Spitzer-identified massive YSOs in a $\sim 1.5 \mathrm{deg}^{2}$ area in the LMC will be presented in Zivkov et al. (2020).

The classification based on the SED fitting was compared with that introduced by Lebzelter et al. (2018) for those AGB candidates for which the Gaia DR2 photometry is available (73 stars), as shown in Fig. 7. Lebzelter et al. (2018) divided between $\mathrm{O}$ - and $\mathrm{C}$-rich stars according to their position in a specific diagram involving the difference between two Wesenheit functions 


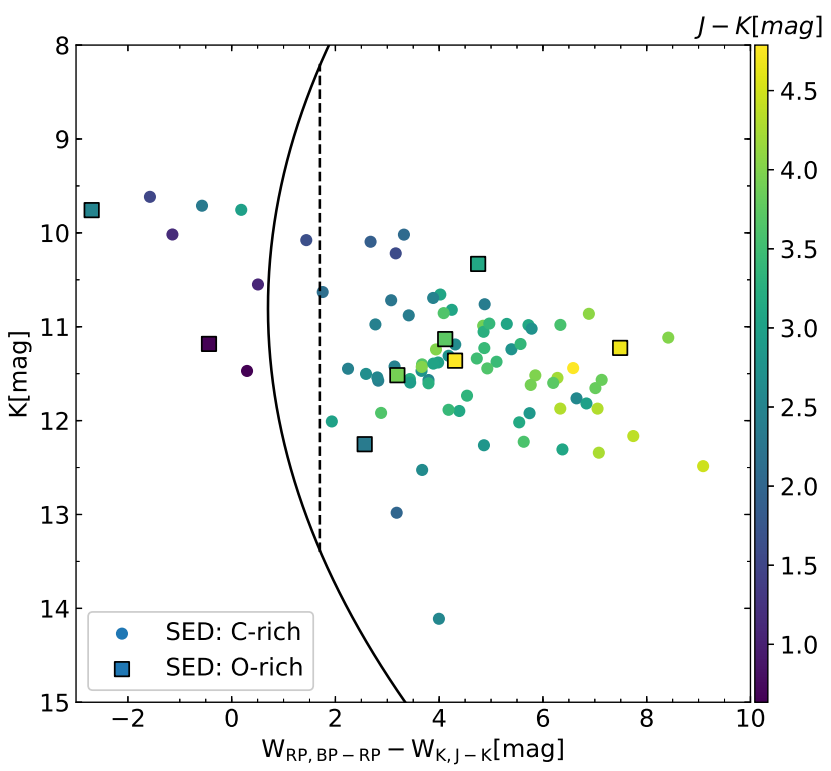

Fig. 7. Classification of the AGB candidates in this work based on the VISTA $K_{s}$ band and Gaia DR2 photometry (Lebzelter et al. 2018). The solid line indicates the separation between oxygen-rich $(\mathrm{O})$ stars (to the left of the curve) and C stars, while the dashed line separates $C$ stars from the most extreme dust-enshrouded stars. All the stars are colourcoded according to their $(J-K)$ colours.

obtained by combining the $J, K_{s}, G_{\mathrm{BP}}$, and $G_{\mathrm{RP}}$ photometric bands, $W_{\mathrm{RP}, \mathrm{BP}-\mathrm{RP}}-W_{K_{s}, J-K_{s}}$ (see the left panel of Fig. 1, and Table A.1 of Lebzelter et al. 2018). The Wesenheit functions are defined as

$W_{K_{s}, J-K_{s}}=K_{s}-0.686 \cdot\left(J-K_{s}\right)$,

where $J$ and $K_{s}$ are the VISTA bands, and:

$W_{\mathrm{RP}, \mathrm{BP}-\mathrm{RP}}=\mathrm{WRP}=G_{\mathrm{RP}}-1.3 \cdot\left(G_{\mathrm{BP}}-G_{\mathrm{RP}}\right)$.

Of the selected stars, 65 sources are classified as C-rich and 8 as O-rich. We find that 72 sources are classified as carbon (C) stars by both methods, while only 6 sources are classified as extreme stars by Lebzelter et al. (2018) are classified as Orich by the SED template fitting developed here. Two stars out of eight that are O-rich for Lebzelter et al. (2018) are classified as O-rich on the basis of the templates. When the classification by Lebzelter et al. (2018) is considered, the SED classification method therefore yields a comparable result for $\mathrm{C}$ stars but disagrees on O-rich stars. Based on the SED fitting classification method, of the 217 AGB candidates, 22 are O-rich, 1 is a PN, and the remainder are $\mathrm{C}$-rich stars.

The AGB candidates were also fitted using the public database of synthetic spectra and photometry for C stars presented in Nanni et al. (2019). These spectra are obtained by consistently computing the growth of dust grains of different species (amorphous carbon and silicon carbide for $\mathrm{C}$ stars) coupled with a stationary dust-driven wind (Nanni et al. 2013, 2014) and a dust radiative transfer code (Groenewegen 2012). The optical constants for amorphous carbon dust are selected to reproduce the photometry in the IR and in the Gaia bands (Nanni et al. 2016; Nanni 2019). We here adopted the data set of Hanner (1988) combined with small grains $<0.04 \mu \mathrm{m}$. The metallicity of the synthetic models is $Z=0.004$ and 0.006 for the SMC and LMC, respectively. As in Nanni et al. (2019), we selected only the models of those that were computed for which the outflow

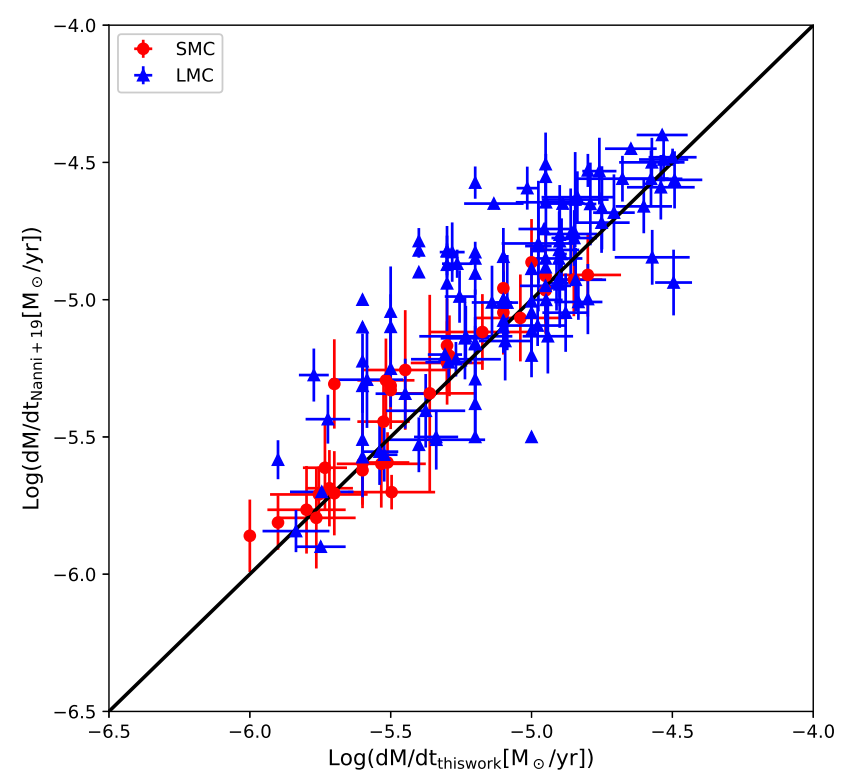

Fig. 8. Comparison between the MLR obtained by Nanni et al. (2019) with the rate we derived for stars in common. The solid black line is the one-to-one relation.

was accelerated through dust-driven wind when the input MLR was $\log (\dot{M}) \geqq-5.5 M_{\odot} \mathrm{yr}^{-1}$. The best fit between the observed and synthetic photometry allows us to estimate the (gas) MLR, the dust production rate, and the luminosity of each star, without the need to assume quantities such as the gas-to-dust ratio and the wind speed of the outflow. The results for the luminosity and MLRs derived from the fitting procedure are provided in Table D.1. In Fig. 8 the MLRs derived in this work and from Nanni et al. (2019) for sources in common are compared. We find that the results agree well, with a scatter of 0.23 dex.

Only 44 and $8 \mathrm{C}$ stars we analysed are missing from the analysis of Nanni et al. (2019) for the LMC and the SMC, respectively. These sources were not included in the catalogues matched by Nanni et al. (2019) (which included C stars from Riebel et al. 2012; Ruffle et al. 2015; Srinivasan et al. 2016; Jones et al. 2017, GS18). The total dust production rate of these missing stars was calculated, and we found that their total contribution is negligible with respect to the total dust production rate of the entire AGB population in these galaxies.

The properties derived for the sources we analysed in this work (period, bolometric luminosity and MLRs) were compared with those derived by Nanni et al. (2019) for the entire population of $\mathrm{C}$ stars in the LMC for which the period has been determined (Riebel et al. 2012). The results are shown in Fig. 9, where we correlate the luminosity with the period, the MLR with the luminosity, and the MLR with the period. The sources we analysed span the entire range of $M_{\mathrm{bol}}$, while the periods are usually between $2.4<\log P(\mathrm{~d})<3.1$. The estimated MLR is in general greater than $\approx 10^{-6} M_{\odot} \mathrm{yr}^{-1}$ and correlates with the luminosity, even though the scatter is large, and with the period.

\section{Discussion}

\subsection{Comparison with periods from Gaia}

Table 1 contains 32 sources that are listed in GDR2 with a "MIRA_SR" classification and a period. The comparison with the period derived from the NIR data in the present paper is excellent; the value of $\left(P_{\text {Gaia }}-P_{\mathrm{NIR}}\right) / \sigma_{\text {Gaia }}$ ranges from -2.2 to 

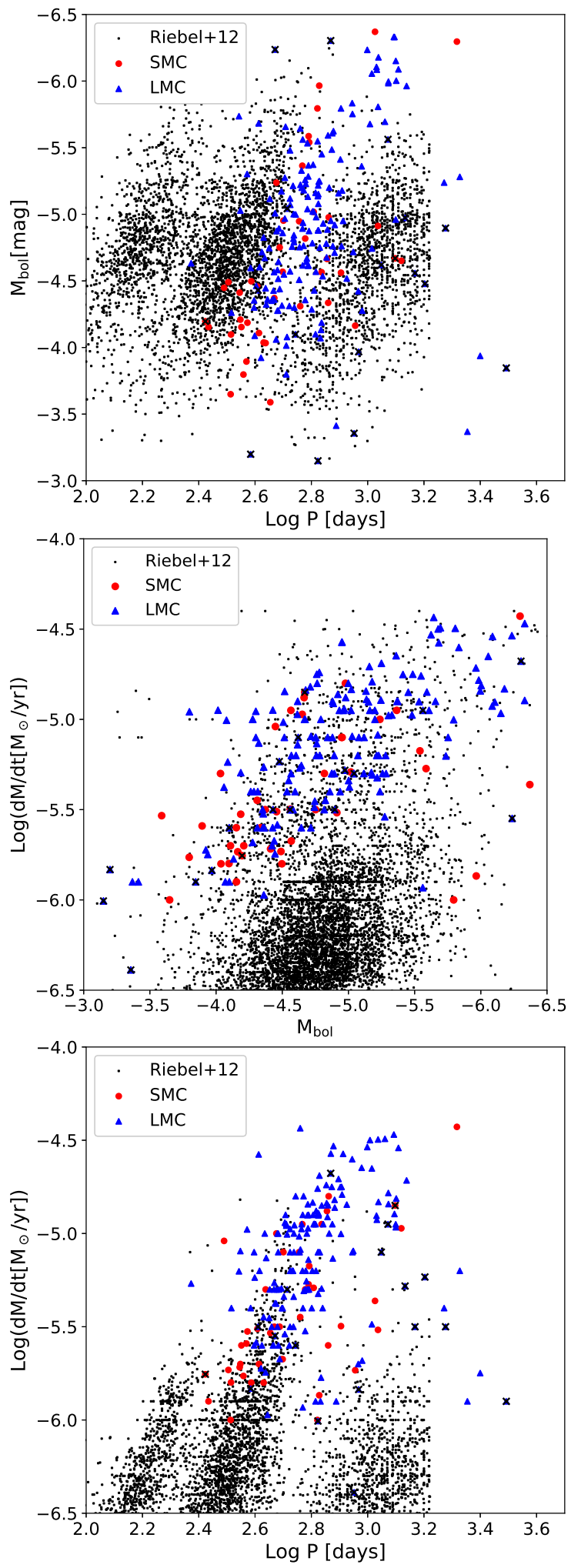

Fig. 9. Top panel: bolometric luminosity vs. period obtained from the SED fitting of the $\mathrm{C}$ stars selected in this work: $\mathrm{C}$ stars in the SMC are indicated with red dots, and C stars in the LMC are plotted with blue triangles. For comparison, we overplot the population of $\mathrm{C}$ stars in the LMC for which the period has been determined from Riebel et al. (2012), which have been fitted by Nanni et al. (2019). To avoid superposition in the plot, $M_{\mathrm{bol}}$ has been changed by a random number between -0.25 and $+0.25 \mathrm{mag}$. The sources for which the period has been flagged with a superscript "a" or " $b$ " in Table C.2 are indicated with a cross. Middle and bottom panels: relations between MLR vs. the bolometric luminosity and MLR vs. period, respectively.
+2.4 with a median value of +0.1 . The periods that are presented here are more precise (the median error in the period is 70 days in the Gaia data and 8 days here), but the error in the period determination by Gaia is expected to decrease significantly with further data releases.

\subsection{Comparison with the IRSF monitoring data in the SMC}

The NIR monitoring data for three stars has recently been published by Ita et al. (2018) using the InfraRed Survey Facility (IRSF, Kato et al. 2007). These data were not used in our LC analysis and can therefore serve as an independent test. The $K$ band data ( $H$ band for $12.627600-72.858307)$ were analysed in the same way as the VMC data and literature data. Table 2 compares the periods and amplitudes. For the first two stars the agreement is good. In the last case the periods do not agree within their mutual error bars, but this is a faint source for the IRSF survey ( $K \sim 15.5 \mathrm{mag}$ ), while our analysis might be hampered by the relatively small number of data points (18 in total).

\subsection{LPVs with periods over 1000 days}

Of the likely AGB stars, 34 stars have periods longer than 1000 days $^{6}$. The longest period we find is $3108 \mathrm{~d}$, but this object has alternative periods that are much shorter. Six other stars also have shorter possible periods, and therefore the periods beyond 1000 days are only tentative in these cases. It will be interesting to analyse the IRSF data for the LMC objects when they become available (cf. the SMC data presented in Ita et al. 2018).

Nine stars with periods over 1000 days in the MCs were previously reported by Wood et al. (1992) and Whitelock et al. (2003) based on NIR observations. Recently, Menzies et al. (2019) presented a compilation of Miras with periods over 1000 days in the Milky Way (17), SMC (3), and LMC (18), while Karambelkar et al. (2019) published a list of 417 luminous IR variables, of which 86 have periods over 1000 days, located in seven galaxies (the majority in M 83 (49 objects), and M 81 (11 objects)). The compilation by Menzies et al. (2019) was based on Wood et al. (1992) and Whitelock et al. (2003), OGLE data in the SMC and LMC (Soszyński et al. 2011, 2009a), and GS18 (which is partly based on a preliminary analysis of VMC data). Of the 21 MC objects, 18 are confirmed with updated periods. Three objects are not in our list. One object (IRAS 05506-7053) is too red; the period in GS18 is based on WISE data. The two others (HV 888 and HV 11417) are too blue to have passed the selection criteria, and both have been classified as RSGs (GS18, Kraemer et al. 2017).

The longest period in the study of GS18 of almost 400 AGB stars with Spitzer IRS spectra is $1810 \mathrm{~d}$ based on a reanalysis of OGLE-III data for MSX SMC 055 (also known as OGLE-SMC-LPV-08137 and IRAS 00483-7347). No errors on the period are given in GS18 because they are very hard to reliably estimate for these long periods. The period in the OGLE-III release is $1859 \mathrm{~d}$, which gives some indication of the uncertainty. In Groenewegen et al. (2009) such a long-period has been recognised based on OGLE-II data and, it was suggested that the object may be a candidate super-AGB star, based on its long period and very high luminosity. Updated parameters were given in GS18, where a current pulsation mass of about $9 M_{\odot}$ was derived. It has a large amplitude $(0.8 \mathrm{mag}$ in $I)$, but its LC is not very regular in shape (see the middle panel of Fig. 7 in Soszyński et al. 2011). Based on the IR LC a period

6 We included one star with a period of $997 \pm 12$ days. 
Table 1. Comparison of periods for sources in common between our study and Gaia DR2.

\begin{tabular}{|c|c|c|c|c|}
\hline $\begin{array}{l}\text { RA } \\
(\mathrm{deg})\end{array}$ & $\begin{array}{c}\text { Dec } \\
(\operatorname{deg})\end{array}$ & GDR2 source id & $\begin{array}{r}\text { Period } \\
\text { Gaia }(\mathrm{d})\end{array}$ & $\begin{array}{r}\text { Period } \\
\text { this work }(\mathrm{d})\end{array}$ \\
\hline 04.876441 & -72.465755 & 4689594530461666432 & $335 \pm 29$ & $265 \pm 1$ \\
\hline 07.572240 & -72.472302 & 4689184000304539520 & $531 \pm 85$ & $508 \pm 12$ \\
\hline 08.511784 & -72.363404 & 4689188428414841216 & $336 \pm 37$ & $356 \pm 1$ \\
\hline 09.660904 & -72.008445 & 4689221379397747968 & $513 \pm 90$ & $385 \pm 8$ \\
\hline 09.828878 & -70.131865 & 4702368897059777792 & $343 \pm 27$ & $320 \pm 4$ \\
\hline 13.053875 & -73.148050 & 4685938821845281280 & $821 \pm 284$ & $796 \pm 5$ \\
\hline 13.292230 & -72.198509 & 4689061778454380416 & $624 \pm 157$ & $491 \pm 56$ \\
\hline 21.130633 & -71.620707 & 4687594377067361280 & $549 \pm 103$ & $724 \pm 12$ \\
\hline 25.642854 & -71.369942 & 4687832356908117120 & $562 \pm 172$ & $545 \pm 23$ \\
\hline 69.714864 & -68.402870 & 4656274857070901760 & $580 \pm 103$ & $513 \pm 2$ \\
\hline 73.110342 & -68.577069 & 4655516060931435008 & $3937 \pm 3845$ & $744 \pm 8$ \\
\hline 73.927019 & -68.281715 & 4661533550631677184 & $466 \pm 62$ & $468 \pm 7$ \\
\hline 74.358023 & -74.020732 & 4649773380396677376 & $437 \pm 50$ & $547 \pm 11$ \\
\hline 74.535830 & -67.878353 & 4661558083486627328 & $433 \pm 53$ & $474 \pm 13$ \\
\hline 74.536373 & -71.966533 & 4654485474923219968 & $443 \pm 73$ & $536 \pm 3$ \\
\hline 74.731098 & -68.218399 & 4661543033920475392 & $612 \pm 70$ & $495 \pm 10$ \\
\hline 76.076144 & -74.117919 & 4649713079054327424 & $450 \pm 58$ & $493 \pm 13$ \\
\hline 77.550727 & -65.326051 & 4663596882956831104 & $664 \pm 68$ & $599 \pm 98$ \\
\hline 78.161438 & -64.203811 & 4664123102346900864 & $723 \pm 216$ & $883 \pm 14$ \\
\hline 79.026012 & -70.825900 & 4651988037356793600 & $515 \pm 122$ & $516 \pm 10$ \\
\hline 79.994330 & -70.471053 & 4651927113213190272 & $512 \pm 56$ & $455 \pm 8$ \\
\hline 80.792265 & -67.835106 & 4658834421384702208 & $593 \pm 95$ & $714 \pm 34$ \\
\hline 80.802424 & -64.158664 & 4661168096128809216 & $435 \pm 43$ & $449 \pm 3$ \\
\hline 81.285698 & -73.278491 & 4651133854242537728 & $321 \pm 25$ & $310 \pm 6$ \\
\hline 82.281667 & -66.970853 & 4660210735084020608 & $495 \pm 54$ & $490 \pm 6$ \\
\hline 82.751418 & -69.179865 & 4658428873394148352 & $645 \pm 174$ & $612 \pm 32$ \\
\hline 83.558539 & -68.978837 & 4658446499944028800 & $550 \pm 249$ & $548 \pm 7$ \\
\hline 83.720398 & -71.006350 & 4657091970360446464 & $710 \pm 121$ & $715 \pm 7$ \\
\hline 83.767073 & -69.954142 & 4657247619989849472 & $532 \pm 61$ & $523 \pm 21$ \\
\hline 85.201902 & -69.560027 & 4657599188763136896 & $1173 \pm 829$ & $576 \pm 5$ \\
\hline 86.488037 & -64.462946 & 4756251319078635648 & $423 \pm 55$ & $388 \pm 9$ \\
\hline 89.223262 & -67.070124 & 4659600643536501504 & $509 \pm 87$ & $414 \pm 35$ \\
\hline
\end{tabular}

Notes. GDR2 does not list periods but a frequency $\omega$ and its error $\sigma$. Period and error are calculated as $1 / \omega$ and $1 / \omega-1 /(\omega+\sigma)$ for simplicity.

Table 2. Comparison with three sources in the IRSF survey.

\begin{tabular}{lccrrrr}
\hline \hline $\begin{array}{l}\text { RA } \\
(\mathrm{deg})\end{array}$ & $\begin{array}{c}\text { Dec } \\
(\mathrm{deg})\end{array}$ & Filter & $\begin{array}{r}\text { Period } \\
(\mathrm{d})\end{array}$ & $\begin{array}{r}\text { Amplitude } \\
(\mathrm{mag})\end{array}$ & $\begin{array}{r}\text { Period } \\
(\mathrm{d})\end{array}$ & $\begin{array}{r}\text { Amplitude } \\
(\mathrm{mag})\end{array}$ \\
\hline & & & IRSF & & \multicolumn{2}{c}{ VMC } \\
12.627600 & -72.858307 & $H$ & $1035.8 \pm 5.3$ & $0.39 \pm 0.04$ & $1062 \pm 10$ & $0.56 \pm 0.05^{(a)}$ \\
14.860309 & -72.394926 & $K$ & $421.9 \pm 1.0$ & $0.54 \pm 0.03$ & $434 \pm 3$ & $0.52 \pm 0.20$ \\
15.173292 & -72.633497 & $K$ & $391.6 \pm 2.4$ & $0.53 \pm 0.14$ & $451 \pm 7$ & $0.37 \pm 0.15$ \\
\hline
\end{tabular}

Notes. ${ }^{(a)}$ In the $K$ band.

of $2075 \pm 15 \mathrm{~d}$ is derived here, about $4 \sigma$ away from the optically determined period considering the mutual error bars.

Our sample of likely AGB stars contains two stars that have very long periods ${ }^{7}$. They are VMC J052454.84-682958.02 (81.228480 -68.499449), with $P=2261 \pm 41 \mathrm{~d}$ and amplitude $0.20 \pm 0.02 \mathrm{mag}$, and VMC J045211.18-701244.83 (73.046582 -70.212454), with $P=2510 \pm 67 \mathrm{~d}$ and amplitude

\footnotetext{
7 Excluding 70.632433-74.796568 with $P=3108 \mathrm{~d}$ because a shorter period might be the correct one as well, and $81.098476-65.536337$ with $P=2125 \mathrm{~d}$ but a rather poor fit and an uncertain amplitude of $0.38 \pm 0.18 \mathrm{mag}$.
}

$0.15 \pm 0.06$ mag. Little is known about the former source. It is not listed in SIMBAD. Its SED is very well fitted by that of a dusty O-rich star, but its luminosity based on the template fitting is low $\left(L \sim 1500 L_{\odot}\right)$ and its amplitude small for a Mira variable. The latter source is listed as a candidate post-AGB object. In ViZieR some catalogues are associated with a source classified as a PN, but often located at about $2^{\prime \prime}$ away. VMC $Y$ - and $K_{s}$-band images were inspected. The finding chart in Morgan (1994) (object 9) suffers from poor resolution and is not clear. A slightly better chart was published by Leisy et al. (1997) but does not allow us to unequivocally distinguish between two sources in the VMC images. The coordinates published there and in 
Reid \& Parker (2006, RP 1607) refer to a source located at about $2.2^{\prime \prime}$ to the SE of the very red VMC source referred to in this paper. In any case, the luminosity based on the template fitting is low $\left(L \sim 3000 L_{\odot}\right)$, and its amplitude small for a Mira variable.

One of the original ideas behind this work was to find stars with very long periods as a tracer of the most massive AGB and super-AGB stars. The nature of the two stars with $2000+$ day periods would be interesting to investigate but they are unlikely to be as massive as the about $9 M_{\odot}$ estimated for MSX SMC 055 (GS18).

\subsection{Bolometric PL-relation}

Figure 10 shows the bolometric PL-relation. The top panel shows all 254 objects for which the template fitting was performed. The likely C-rich objects (i.e. listed as " $\mathrm{C}$ " in Col. 10 in Table C.2) are plotted as open squares, the likely O-rich objects (i.e. listed as "O" in Table C.2) are plotted as open triangles, the other AGB stars (i.e. listed as O(AGB), O(OTH), and $\mathrm{C}(\mathrm{AGB})$ in Table C.2) as filled circles. The 37 likely nonAGB stars are plotted as dots. The likely C-rich Miras (with an amplitude $>0.2 \mathrm{mag}$ ) are plotted in the middle panel. The blue dashed line is a fit to the data, excluding seven outliers (plotted as dots) based on iterative $3 \sigma$ clipping. The fit is $M_{\mathrm{bol}}=$ $(-2.27 \pm 0.20) \cdot \log P+(1.45 \pm 0.54) \mathrm{mag}$ using 182 stars and with an rms of $0.41 \mathrm{mag}$ using a linear bi-sector fit (using the code SIXLIN from Isobe et al. 1990). When we restrict the sample to LMC objects (144 objects) or eliminate the objects for which an alternative period cannot be excluded, the coefficients of the fit or the rms value do not change significantly.

The likely O-rich Miras (with an amplitude $>0.2 \mathrm{mag}$ ) are plotted in the bottom panel. The blue dashed line is a fit to the data, excluding two outliers (plotted as dots) based on iterative $3 \sigma$ clipping. The fit is $M_{\mathrm{bol}}=(-2.97 \pm 0.09) \cdot \log P+(2.59 \pm$ $0.28)$ mag based on 11 stars and with an rms of 0.36 mag. The red dotted lines in the two panels indicate relations from Feast et al. (1989) for C- and O-rich Miras in the LMC respectively. These relations were derived for $P<420 \mathrm{~d}$ and were shifted using an LMC distance modulus of 18.477 mag from Pietrzyński et al. (2019).

\section{Summary}

Based on CCD and CMD diagrams and the properties of known Mira variables detected by the OGLE survey, we selected a sample of 1299 sources as (candidate) LPVs with periods longer than 450 days. Multiple VMC $K_{s}$-band data were combined with literature data to construct LCs. A limitation is that magnitudes from different $K$-type filters are mixed because it is almost impossible to uniformly and consistently convert all data into a uniform system. The LCs were analysed for periodic variations. Mean magnitudes are published for all stars, and periods and amplitudes for 949 stars. Based on the pulsation properties of known OGLE Miras and when we remove all stars with a known period (except for those longer than 1000 days), a sample of 254 stars was retained for further study. The SEDs of these stars were derived and fitted with template distributions of known objects. A final list of 217 candidate LPVs was obtained. Thirty-four objects have periods longer than 1000 days, but some have a viable alternative period that is shorter. The Mira with the longest known period in the MCs from OGLE data (with $P=1810 \mathrm{~d}$ ) was derived to have a period of $2075 \mathrm{~d}$ based on its IR LC. Two stars were found to have longer periods, but the luminosities and pulsation amplitudes of both are unlike those of Miras. The
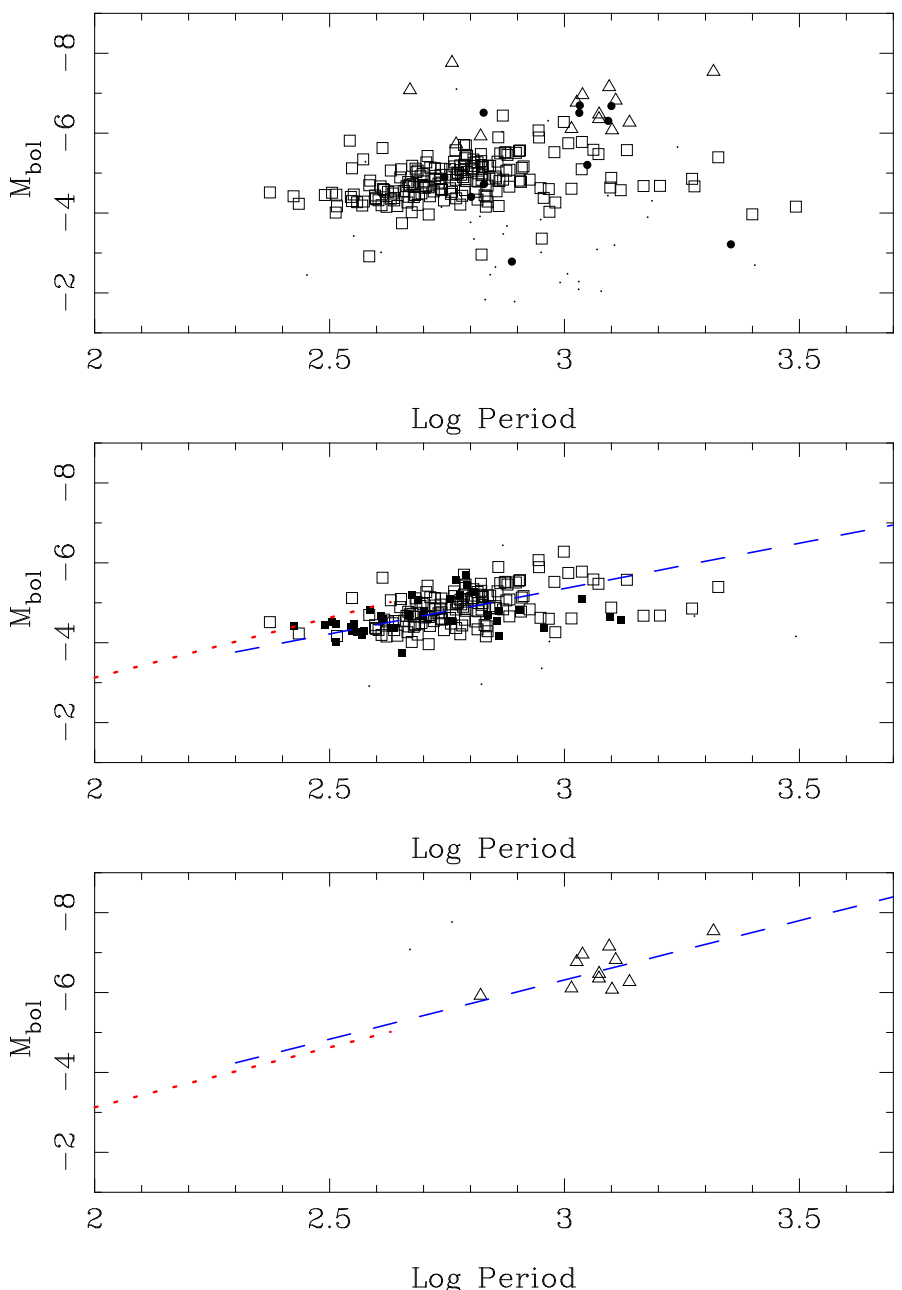

Fig. 10. Bolometric PL-relations for all the 254 objects for which the template fitting was performed (top panel), and the subsets of likely Crich Miras (middle panel), and O-rich Miras (bottom panel). The likely C-rich objects are plotted as open squares, the likely O-rich objects are plotted as open triangles, and the other AGB stars as filled circles, see the main text for details. The 37 likely non-AGB stars are plotted as dots. The likely C-rich Miras (with an amplitude $>0.2 \mathrm{mag}$ ) are plotted in the middle panel. The objects in the LMC are plotted with open symbols, and those in the SMC with filled symbols. The blue dashed line is a fit to the data, excluding outliers (plotted as dots). The likely O-rich Miras (with an amplitude $>0.2 \mathrm{mag}$ ) are plotted in the bottom panel. The blue dashed line is a fit to the data, excluding outliers (plotted as dots). The red dotted lines in the two panels indicate relations from the literature derived at shorter periods, see the main text for details.

bolometric magnitude - period relation was derived for both likely $\mathrm{O}$ - and $\mathrm{C}$-rich stars in the sample.

Acknowledgements. We thank the Cambridge Astronomy Survey Unit (CASU) and the Wide Field Astronomy Unit (WFAU) in Edinburgh for providing calibrated data products under the support of the Science and Technology Facility Council (STFC) in the UK. M.-R.L.C. acknowledges support from the European Research Council (ERC) under the European Union's Horizon 2020 research and innovation programme (Grant agreement No 682115). A.N. acknowledges support from the Centre National d'Etudes Spatiale (CNES) through a post-doctora fellowship. This publication is partly based on the OGLE observations obtained with the Warsaw Telescope at the Las Campanas Observatory, Chile, operated by the Carnegie Institution of Washington. This paper utilises public domain data originally obtained by the MACHO Project, whose work was performed under the joint auspices of the U.S. Department of Energy, National Nuclear Security Administration by the University of California, Lawrence Livermore National Laboratory under contract No. W-7405-Eng-48, the National Science Foundation through the Center for Particle Astrophysics of the University of California 
A\&A 636, A48 (2020)

under cooperative agreement AST-8809616, and the Mount Stromlo and Siding Spring Observatory, part of the Australian National University. This publication makes use of data products from the Wide-field Infrared Survey Explorer, which is a joint project of the University of California, Los Angeles, and the Jet Propulsion Laboratory/California Institute of Technology, funded by the National Aeronautics and Space Administration. This research has made use of the SIMBAD database and the VizieR catalogue access tool, both operated at CDS, Strasbourg, France. This work has made use of data from the European Space Agency (ESA) mission Gaia (https://www. cosmos.esa.int/gaia), processed by the Gaia Data Processing and Analysis Consortium (DPAC, https://www. cosmos.esa.int/web/gaia/dpac/consortium). Funding for the DPAC has been provided by national institutions, in particular the institutions participating in the Gaia Multilateral Agreement.

\section{References}

Aringer, B., Girardi, L., Nowotny, W., Marigo, P., \& Lederer, M. T. 2009, A\&A, 503,913

Aringer, B., Marigo, P., Nowotny, W., et al. 2019, MNRAS, 487, 2133

Bessell, M. S., \& Brett, J. M. 1989, in JHKLM Photometry: Standard Systems, Passbands and Intrinsic Colors, ed. E. F. Milone, 341, 61

Bolatto, A. D., Simon, J. D., Stanimirović, S., et al. 2007, ApJ, 655, 212

Boyer, M. L., Srinivasan, S., van Loon, J. T., et al. 2011, AJ, 142, 103

Carpenter, J. M. 2001, AJ, 121, 2851

Cioni, M.-R. L., Clementini, G., Girardi, L., et al. 2011, A\&A, 527, A116

Cioni, M.-R. L., Ripepi, V., Clementini, G., et al. 2017, Eur. Phys. J. Web Conf., 152,01008

Cross, N. J. G., Collins, R. S., Mann, R. G., et al. 2012, A\&A, 548, A119

Cutri, R. M., Skrutskie, M. F., van Dyk, S., et al. 2003, VizieR Online Data Catalog: II/246

Cutri, R. M., Skrutskie, M. F., van Dyk, S., et al. 2012, VizieR Online Data Catalog: II/281

Cutri, R. M., Wright, E. L., Conrow, T., et al. 2013, VizieR Online Data Catalog: II $/ 328$

DENIS Consortium 2005, VizieR Online Data Catalog: II/263

Emerson, J., \& Sutherland, W. 2010, The Messenger, 139, 2

Feast, M. W., Glass, I. S., Whitelock, P. A., \& Catchpole, R. M. 1989, MNRAS, 241,375

Ferraro, F. R., Fusi Pecci, F., Testa, V., et al. 1995, MNRAS, 272, 391

Fraser, O. J., Hawley, S. L., \& Cook, K. H. 2008, AJ, 136, 1242

Frogel, J. A., Mould, J., \& Blanco, V. M. 1990, ApJ, 352, 96

Gaia Collaboration (Brown, A. G. A., et al.) 2018, A\&A, 616, A1

Glass, I. S., \& Evans, T. L. 1981, Nature, 291, 303

Glass, I. S., \& Reid, N. 1985, MNRAS, 214, 405

Gordon, K. D., Meixner, M., Meade, M. R., et al. 2011, AJ, 142, 102

Groenewegen, M. A. T. 2004, A\&A, 425, 595

Groenewegen, M. A. T. 2012, A\&A, 543, A36

Groenewegen, M. A. T., \& Blommaert, J. A. D. L. 1998, A\&A, 332, 25

Groenewegen, M. A. T., \& Sloan, G. C. 2018, A\&A, 609, A114

Groenewegen, M. A. T., Sevenster, M., Spoon, H. W. W., \& Pérez, I. 2002, A\&A, 390,511

Groenewegen, M. A. T., Sloan, G. C., Soszyński, I., \& Petersen, E. A. 2009, A\&A, 506, 1277

Groenewegen, M. A. T., Vlemmings, W. H. T., Marigo, P., et al. 2016, A\&A 596, A50

Gruendl, R. A., \& Chu, Y.-H. 2009, ApJS, 184, 172

Gullieuszik, M., Groenewegen, M. A. T., Cioni, M.-R. L., et al. 2012, A\&A, 537, A 105

Hanner, M. 1988, Grain Optical Properties, Tech. rep., 22

Hauschildt, P. H., Allard, F., \& Baron, E. 1999, ApJ, 512, 377

Höfner, S., \& Olofsson, H. 2018, A\&ARv, 26, 1

Holl, B., Audard, M., Nienartowicz, K., et al. 2018, A\&A, 618, A30

Houck, J. R., Roellig, T. L., van Cleve, J., et al. 2004, ApJS, 154, 18

Huang, C. D., Riess, A. G., Hoffmann, S. L., et al. 2018, ApJ, 857, 67

Huang, C. D., Riess, A. G., Yuan, W., et al. 2020, ApJ, 889, 5

Hughes, S. M. G. 1989, AJ, 97, 1634

Hughes, S. M. G., \& Wood, P. R. 1990, AJ, 99, 784

Irwin, M. J. 2009, UKIRT Newsletter, 25, 15

Isobe, T., Feigelson, E. D., Akritas, M. G., \& Babu, G. J. 1990, ApJ, 364, 104

Ita, Y., Tanabé, T., Matsunaga, N., et al. 2004, MNRAS, 347, 720

Ita, Y., Matsunaga, N., Tanabé, T., et al. 2018, MNRAS, 481, 4206

Ivezić, Ž., Nenkova, M., \& Elitzur, M. 1999, Astrophysics Source Code Library [record ascl:9911.0Q1]

Jones, O. C., Woods, P. M., Kemper, F., et al. 2017, MNRAS, 470, 3250

Kamath, D., Wood, P. R., Soszyński, I., \& Lebzelter, T. 2010, MNRAS, 408, 522

Karambelkar, V. R., Adams, S. M., Whitelock, P. A., et al. 2019, ApJ, 877, 110

Kastner, J. H., Thorndike, S. L., Romanczyk, P. A., et al. 2008, AJ, 136, 1221
Kato, D., Nagashima, C., Nagayama, T., et al. 2007, PASJ, 59, 615

Kim, D.-W., Protopapas, P., Bailer-Jones, C. A. L., et al. 2014, A\&A, 566, A43 Koen, C., Marang, F., Kilkenny, D., \& Jacobs, C. 2007, MNRAS, 380, 1433

Kraemer, K. E., Sloan, G. C., Wood, P. R., Jones, O. C., \& Egan, M. P. 2017, ApJ, 834, 185

Lebouteiller, V., Barry, D. J., Spoon, H. W. W., et al. 2011, ApJS, 196, 8 Lebzelter, T., Mowlavi, N., Marigo, P., et al. 2018, A\&A, 616, L13

Leisy, P., Dennefeld, M., Alard, C., \& Guibert, J. 1997, A\&AS, 121, 407

Lenz, P., \& Breger, M. 2005, Commun. Asteroseismol., 146, 53

Meixner, M., Gordon, K. D., Indebetouw, R., et al. 2006, AJ, 132, 2268

Menzies, J. W., Whitelock, P. A., Feast, M. W., \& Matsunaga, N. 2019, MNRAS, 483, 5150

Morgan, D. H. 1994, A\&AS, 103, 235

Muraveva, T., Subramanian, S., Clementini, G., et al. 2018, MNRAS, 473, 3131

Nanni, A. 2019, MNRAS, 482, 4726

Nanni, A., Bressan, A., Marigo, P., \& Girardi, L. 2013, MNRAS, 434, 2390

Nanni, A., Bressan, A., Marigo, P., \& Girardi, L. 2014, MNRAS, 438, 2328

Nanni, A., Marigo, P., Groenewegen, M. A. T., et al. 2016, MNRAS, 462, 1215

Nanni, A., Marigo, P., Girardi, L., et al. 2018, MNRAS, 473, 5492

Nanni, A., Groenewegen, M. A. T., Aringer, B., et al. 2019, MNRAS, 487, 502

Nishida, S., Tanabé, T., Nakada, Y., et al. 2000, MNRAS, 313, 136

Oliveira, J. M., van Loon, J. T., Chen, C. H. R., et al. 2009, ApJ, 707, 1269

Payne-Gaposchkin, C. H. 1971, Smithsonian Contributions to Astrophysics

(Washington: Smithsonian Institution Press), 13

Pietrzyński, G., Graczyk, D., Gallenne, A., et al. 2019, Nature, 567, 200

Pojmanski, G. 2002, Acta Astron., 52, 397

Press, W. H., Teukolsky, S. A., Vetterling, W. T., \& Flannery, B. P. 1992, Numerical Recipes in FORTRAN. The Art of Scientific Computing (Cambridge: Cambridge University Press)

Ramstedt, S., \& Olofsson, H. 2014, A\&A, 566, A145

Reid, N. 1991, ApJ, 382, 143

Reid, W. A. 2014, MNRAS, 438, 2642

Reid, W. A., \& Parker, Q. A. 2006, MNRAS, 373, 52

Reid, N., Tinney, C., \& Mould, J. 1990, ApJ, 348, 98

Reid, I. N., Hughes, S. M. G., \& Glass, I. S. 1995, MNRAS, 275, 331

Riebel, D., Srinivasan, S., Sargent, B., \& Meixner, M. 2012, ApJ, 753, 71

Ripepi, V., Moretti, M. I., Marconi, M., et al. 2012, MNRAS, 424, 1807

Ripepi, V., Moretti, M. I., Marconi, M., et al. 2015, MNRAS, 446, 3034

Ripepi, V., Marconi, M., Moretti, M. I., et al. 2016, ApJS, 224, 21

Ripepi, V., Cioni, M.-R. L., Moretti, M. I., et al. 2017, MNRAS, 472, 808

Ruffle, P. M. E., Kemper, F., Jones, O. C., et al. 2015, MNRAS, 451, 3504

Sanduleak, N., MacConnell, D. J., \& Philip, A. G. D. 1978, PASP, 90, 621

Seale, J. P., Looney, L. W., Chu, Y.-H., et al. 2009, ApJ, 699, 150

Skiff, B. A. 2014, VizieR Online Data Catalog: 1

Sloan, G. C., Kraemer, K. E., McDonald, I., et al. 2016, ApJ, 826, 44

Soszyński, I., Dziembowski, W. A., Udalski, A., et al. 2007, Acta Astron., 57, 201

Soszyński, I., Poleski, R., Udalski, A., et al. 2008a, Acta Astron., 58, 163 Soszyński, I., Udalski, A., Szymański, M. K., et al. 2008b, Acta Astron., 58, 293 Soszyński, I., Udalski, A., Szymański, M. K., et al. 2009a, Acta Astron., 59, 239 Soszyński, I., Udalski, A., Szymański, M. K., et al. 2009b, Acta Astron., 59, 335 Soszyński, I., Udalski, A., Szymański, M. K., et al. 2010, Acta Astron., 60, 91 Soszyński, I., Udalski, A., Szymański, M. K., et al. 2011, Acta Astron., 61, 217 Soszyński, I., Udalski, A., Poleski, R., et al. 2012, Acta Astron., 62, 219

Spano, M., Mowlavi, N., Eyer, L., et al. 2011, A\&A, 536, A60 Srinivasan, S., Boyer, M. L., Kemper, F., et al. 2016, MNRAS, 457, 2814 Tanabé, T., Nishida, S., Matsumoto, S., et al. 1997, Nature, 385, 509 Trabucchi, M., Wood, P. R., Montalbán, J., et al. 2017, ApJ, 847, 139 Ulaczyk, K., Szymański, M. K., Udalski, A., et al. 2013, Acta Astron., 63, 159 van Loon, J. T., Zijlstra, A. A., Whitelock, P. A., et al. 1997, A\&A, 325, 585

Westerlund, B. E., \& Smith, L. F. 1964, MNRAS, 128, 311

Whitelock, P. A., Feast, M. W., Menzies, J. W., \& Catchpole, R. M. 1989 , MNRAS, 238, 769

Whitelock, P. A., Feast, M. W., van Loon, J. T., \& Zijlstra, A. A. 2003, MNRAS, 342, 86

Whitney, B. A., Sewilo, M., Indebetouw, R., et al. 2008, AJ, 136, 18

Wood, P. R. 1998, A\&A, 338, 592

Wood, P. R. 2000, PASA, 17, 18

Wood, P. R., Bessell, M. S., \& Fox, M. W. 1983, ApJ, 272, 99

Wood, P. R., Whiteoak, J. B., Hughes, S. M. G., et al. 1992, ApJ, 397, 552

Wood, P. R., Alcock, C., Allsman, R. A., et al. 1999, in Asymptotic Giant Branch Stars, eds. T. Le Bertre, A. Lèbre, \& C. Waelkens, IAU Symp., 191, 151 Woods, P. M., Oliveira, J. M., Kemper, F., et al. 2011, MNRAS, 411, 1597 Zickgraf, F. J., Wolf, B., Stahl, O., Leitherer, C., \& Appenzeller, I. 1986, A\&A, 163,119

Zickgraf, F. J., Wolf, B., Stahl, O., \& Humphreys, R. M. 1989, A\&A, 220, 206 Zijlstra, A. A., Loup, C., Waters, L. B. F. M., et al. 1996, MNRAS, 279, 32 Zivkov, V., Oliveira, J. M., Petr-Gotzens, M. G., et al. 2020, MNRAS, 494, 458 


\section{Appendix A: Sample, literature, and period analysis}

Tables A.1-A.3 provide the first entries of the tables that are available at the CDS. They provide information about the 1299 sources for which the $K$-band LCs were analysed. Table A.1 provides basic information about the sample, Table A.2 provides information on the known periodicity from the literature, and
Table A.3 provides the results of the LC analysis. The meaning of the columns is explained in the footnotes to the tables.

Figure A.1 shows examples of the LC fitting. The complete set for the 1299 objects is available at https://zenodo.org/ record/3714889. The model is represented by the (red) solid line. At the top of the plot, the identifier and the period (in days) are listed.

Table A.1. Sample for which a period analysis and LC fitting was performed.

\begin{tabular}{|c|c|c|c|c|c|c|}
\hline $\begin{array}{l}\text { RA } \\
\text { (deg) }\end{array}$ & $\begin{array}{c}\text { Dec } \\
(\operatorname{deg})\end{array}$ & Names & Object type & Spectral type & Gaia type & Spitzer type \\
\hline 4.876441 & -72.465755 & 2MASS J00193036-7227567 & Can. AGB & & MIRA_SR & \\
\hline 5.993721 & -73.631895 & OGLE SMC-LPV-190, 2MASS J00235849-7337548 & Mira & & & \\
\hline 6.088240 & -72.107443 & & & & & \\
\hline 6.498306 & -73.895774 & OGLE SMC-LPV-367 2MASS J00255959-7353448 & Mira & & & \\
\hline 6.795538 & -73.408413 & OGLE SMC-LPV-486 2MASS J00271093-7324303 & Mira & & & \\
\hline 7.329711 & -71.063852 & IRAS F00271-7120 2MASS J00291916-7103499 & $\mathrm{C}$ & $\mathrm{C}$ & & \\
\hline 7.572240 & -72.472302 & OGLE SMC-LPV-881 2MASS J00301737-7228202 & Mira & & MIRA_SR & \\
\hline 7.669748 & -73.712530 & OGLE SMC-LPV-929 2MASS J00304075-7342450 & Mira & & & \\
\hline 7.917060 & -73.798217 & & & & & \\
\hline 7.987056 & -73.520435 & & & & & \\
\hline 8.051002 & -74.801395 & OGLE SMC-LPV-1168 2MASS J00321227-7448049 & Mira & & & \\
\hline 8.117008 & -71.789106 & 2MASS J00322809-7147207 & Can. AGB & & & \\
\hline 8.442051 & -72.749582 & 2MASS J00334612-7244584 & Can. AGB & & & \\
\hline 8.511784 & -72.363404 & 2MASS J00340283-7221482 & Can. AGB & & MIRA_SR & \\
\hline 8.816105 & -73.424329 & OGLE SMC-LPV-1690 2MASS J00351587-7325275 & Mira & & & \\
\hline 8.931123 & -73.352948 & LIN 19 2MASS J00354347-7321106 & Em & & & \\
\hline 8.955451 & -74.127210 & 2MASS J00354932-7407381 & Star & & & \\
\hline 9.108186 & -73.432101 & & & & & \\
\hline 9.164485 & -72.274068 & 2MASS J00363946-7216266 & Can. AGB & & & \\
\hline 9.193053 & -73.526474 & MSX SMC 029 2MASS J00364631-7331351 & PAGB & $\mathrm{C}$ & & C (1) CPAGB (2) CPAGB (3) \\
\hline 9.230702 & -74.741285 & & & & & \\
\hline 9.236272 & -72.421530 & MSX SMC 091 2MASS J00365671-7225175 & $\mathrm{C}$ & & & C (1) CAGB (2) CAGB (3) \\
\hline 9.246025 & -71.636344 & [FBR2002] J003659-713813 & Radio & & & \\
\hline 9.328809 & -72.284239 & 2MASS J00371893-7217031 & Can. AGB & & & \\
\hline 9.384601 & -73.506030 & OGLE J003732.32-733021.3 2MASS J00373232-7330216 & LPV & & & \\
\hline 9.387759 & -72.879192 & 2MASS J00373306-7252451 & Can. AGB & & & \\
\hline 9.446274 & -73.650656 & RAW 21 2MASS J00374710-7339022 & $\mathrm{C}$ & $\mathrm{C}$ & & \\
\hline 9.466017 & -69.829967 & & & & & \\
\hline 9.491884 & -75.208617 & [KID97] C0036-7529 2MASS J00375807-7512309 & $\mathrm{C}$ & & & \\
\hline 9.507699 & -73.790561 & OGLE J003801.87-734725.7 2MASS J00380186-7347259 & Mira & & & \\
\hline 9.614211 & -74.260202 & OGLE SMC-LPV-2488 2MASS J00382740-7415366 & Mira & & & \\
\hline 9.660904 & -72.008445 & 2MASS J00383862-7200304 & Can. AGB & & MIRA_SR & \\
\hline 9.794922 & -71.569425 & & & & & \\
\hline 9.828878 & -70.131865 & [MH95] 414 2MASS J00391894-7007546 & $\mathrm{C}$ & $\mathrm{C}$ & MIRA_SR & \\
\hline 9.887294 & -70.295891 & & & & & \\
\hline 10.067795 & -73.020987 & OGLE J004016.25-730115.1 2MASS J00401627-7301156 & Mira & & & \\
\hline 10.137255 & -73.477785 & OGLE J004032.93-732839.7 2MASS J00403293-7328399 & Mira & & & \\
\hline 10.147153 & -73.324740 & OGLE J004035.31-731928.7 2MASS J00403531-7319291 & Mira & & & \\
\hline
\end{tabular}

Notes. Column 1 lists the coordinates (right ascension and declination) of the source in decimal degrees. Column 2 gives some names, as listed by the SIMBAD database. A "?" indicates the name of a source that is located close to the nominal position. Column 3 gives the object type, as listed by the SIMBAD database (As of January 2019. As the SIMBAD database is continuously updated this may change in time). Column 4 gives the spectral type(s), as listed in Skiff (2014). In case of many entries only a selection is listed. Column 5 gives the variable star classification as listed in the GDR2 Gaia Collaboration (2018), Holl et al. (2018). Column 6 gives the classification based on the MIR Spitzer IRS spectra. The full table is available at the CDS.

References. (1) Groenewegen \& Sloan (2018); (2) Kraemer et al. (2017); (3) Ruffle et al. (2015); (4) Jones et al. (2017); (5) Seale et al. (2009); (6) Sloan et al. (2016) (all stars listed as "C" in Groenewegen \& Sloan 2018, also listed in Sloan et al. 2016); (7) Woods et al. (2011). The classifications are taken directly from those papers. For the exact meaning of the abbreviations see the original papers. 


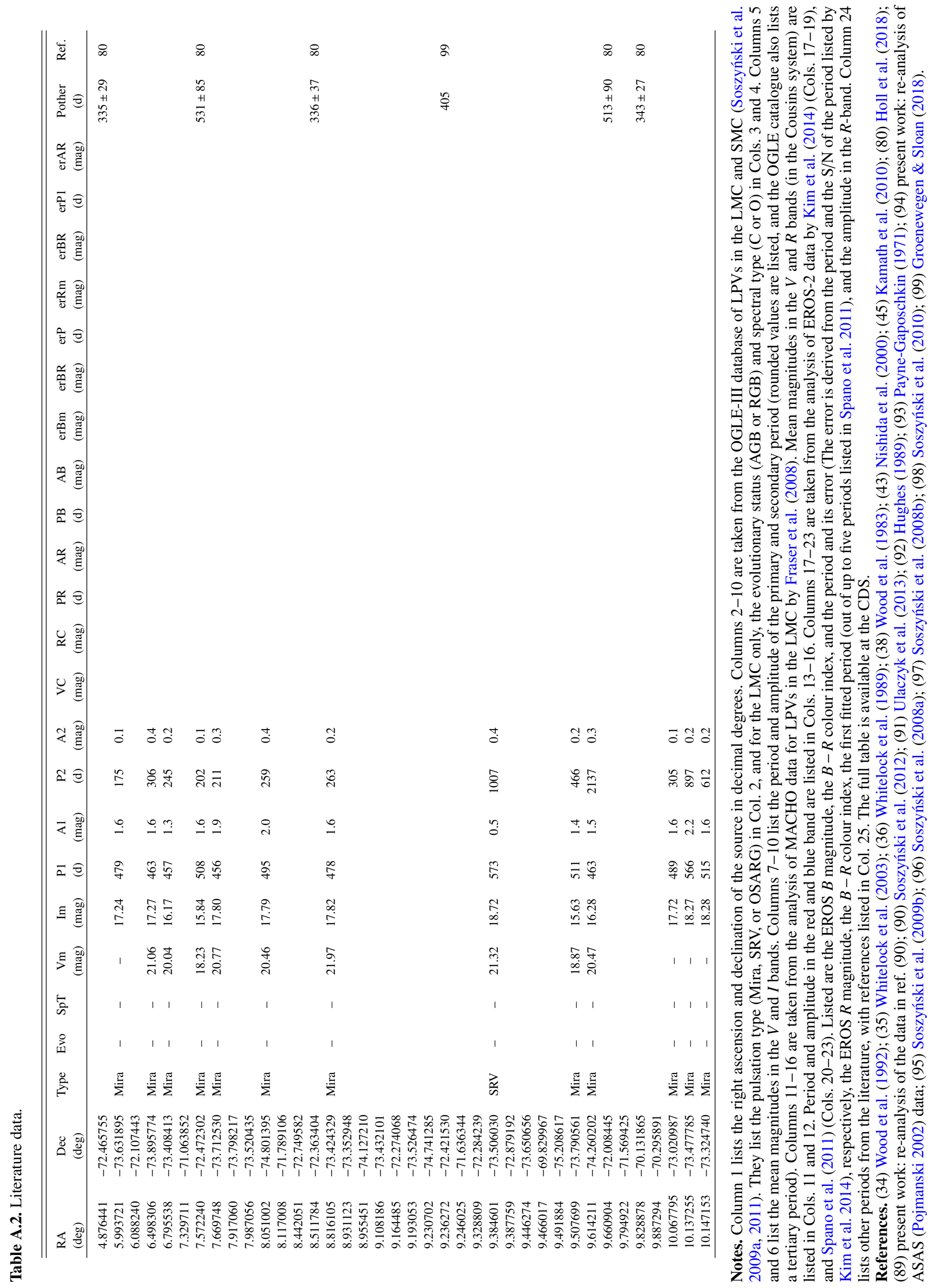


Table A.3. Results from the period analysis.

\begin{tabular}{|c|c|c|c|c|c|c|c|c|c|}
\hline $\begin{array}{l}\text { RA } \\
\text { (deg) }\end{array}$ & $\begin{array}{c}\text { Dec } \\
(\mathrm{deg})\end{array}$ & $\chi_{\mathrm{r}}^{2}$ & $\begin{array}{l}K \\
\text { (mag) }\end{array}$ & $\begin{array}{l}\text { Period } \\
\text { (d) }\end{array}$ & $\begin{array}{l}\text { Amplitude } \\
(\mathrm{mag})\end{array}$ & Data & References & $\begin{array}{l}P_{\text {ini }} \\
(\mathrm{d})\end{array}$ & Remarks \\
\hline 4.876441 & -72.465755 & 19.8 & $11.982 \pm 0.011$ & $265 \pm 1$ & $0.24 \pm 0.05$ & 3236 & 1101113 & & Palt $=333$ \\
\hline 5.993721 & -73.631895 & 308.8 & $11.473 \pm 0.077$ & $479 \pm 6$ & $0.42 \pm 0.08$ & 1418 & 1101113 & 479 & \\
\hline 6.088240 & -72.107443 & 6.7 & $16.452 \pm 0.054$ & & & 1010 & 1 & & \\
\hline 6.498306 & -73.895774 & 268.6 & $11.427 \pm 0.081$ & $468 \pm 5$ & $0.55 \pm 0.18$ & 1418 & 110111213 & 463 & \\
\hline 6.795538 & -73.408413 & 42.2 & $11.455 \pm 0.025$ & $451 \pm 5$ & $0.32 \pm 0.12$ & 1518 & 11113 & 457 & \\
\hline 7.329711 & -71.063852 & 863.7 & $13.422 \pm 0.227$ & $343 \pm 4$ & $0.73 \pm 0.38$ & 1824 & 1101113 & & Palt $=953$ \\
\hline 7.572240 & -72.472302 & 695.6 & $10.792 \pm 0.062$ & $508 \pm 12$ & $0.39 \pm 0.18$ & 3133 & 11011 & 508 & \\
\hline 7.669748 & -73.712530 & 1317.8 & $11.524 \pm 0.154$ & $458 \pm 15$ & $0.62 \pm 0.52$ & 1418 & 110111213 & 456 & \\
\hline 7.917060 & -73.798217 & 4.8 & $14.382 \pm 0.008$ & $740 \pm 14$ & $0.09 \pm 0.05$ & 1418 & 1101112 & & \\
\hline 7.987056 & -73.520435 & 1.3 & $14.786 \pm 0.003$ & & & 1519 & 1101112 & & \\
\hline 8.051002 & -74.801395 & 48.8 & $11.475 \pm 0.038$ & $491 \pm 5$ & $0.29 \pm 0.15$ & 1318 & 1101113 & 495 & \\
\hline 8.117008 & -71.789106 & 70.3 & $11.278 \pm 0.023$ & $600 \pm 4$ & $0.63 \pm 0.07$ & 1721 & 1101113 & & \\
\hline 8.442051 & -72.749582 & 175.6 & $12.980 \pm 0.037$ & $571 \pm 10$ & $0.54 \pm 0.30$ & 1520 & 110111213 & & \\
\hline 8.511784 & -72.363404 & 30.2 & $11.622 \pm 0.025$ & $356 \pm 1$ & $0.70 \pm 0.04$ & 1723 & 1101113 & & \\
\hline 8.816105 & -73.424329 & 119.3 & $11.139 \pm 0.039$ & $482 \pm 5$ & $0.46 \pm 0.07$ & 1622 & 110111213 & 478 & \\
\hline 8.931123 & -73.352948 & 37.8 & $13.720 \pm 0.016$ & & & 1623 & 110111213 & & \\
\hline 8.955451 & -74.127210 & 1.6 & $14.680 \pm 0.003$ & & & 1822 & 11011 & & \\
\hline 9.108186 & -73.432101 & 7.5 & $14.453 \pm 0.008$ & & & 1619 & 1101112 & & \\
\hline 9.164485 & -72.274068 & 19.7 & $12.326 \pm 0.011$ & $466 \pm 2$ & $0.43 \pm 0.05$ & 1922 & 1101113 & & \\
\hline 9.193053 & -73.526474 & 24.7 & $13.372 \pm 0.013$ & & & 1620 & 110111213 & & \\
\hline 9.230702 & -74.741285 & 10.9 & $14.856 \pm 0.016$ & $392 \pm 11$ & $0.05 \pm 0.04$ & 3336 & 11011 & & \\
\hline 9.236272 & -72.421530 & 78.7 & $11.501 \pm 0.025$ & $406 \pm 2$ & $0.52 \pm 0.05$ & 1923 & 1101113 & 405 & \\
\hline 9.246025 & -71.636344 & 1.7 & $14.671 \pm 0.004$ & $960 \pm 39$ & $0.05 \pm 0.03$ & 1921 & 11011 & & \\
\hline 9.328809 & -72.284239 & 5.1 & $11.619 \pm 0.010$ & $362 \pm 1$ & $0.41 \pm 0.01$ & 1923 & 1101113 & & \\
\hline 9.384601 & -73.506030 & 180.6 & $13.187 \pm 0.046$ & $571 \pm 16$ & $0.42 \pm 0.33$ & 1620 & 110111213 & 573 & \\
\hline 9.387759 & -72.879192 & 28.0 & $13.536 \pm 0.016$ & $686 \pm 5$ & $0.67 \pm 0.06$ & 1619 & 1101112 & & \\
\hline 9.446274 & -73.650656 & 605.8 & $13.146 \pm 0.071$ & $992 \pm 12$ & $1.19 \pm 0.19$ & 3439 & 110111213 & & \\
\hline 9.466017 & -69.829967 & 20.3 & $12.504 \pm 0.027$ & $371 \pm 2$ & $0.55 \pm 0.05$ & 1722 & 1101113 & & \\
\hline 9.491884 & -75.208617 & 432.8 & $11.599 \pm 0.098$ & $305 \pm 3$ & $0.41 \pm 0.20$ & 1519 & 1101113 & & \\
\hline 9.507699 & -73.790561 & 226.4 & $10.818 \pm 0.048$ & $505 \pm 11$ & $0.31 \pm 0.19$ & 1822 & 1101113 & 511 & \\
\hline 9.614211 & -74.260202 & 70.8 & $11.272 \pm 0.033$ & $448 \pm 2$ & $0.49 \pm 0.12$ & 1821 & 11013 & 463 & \\
\hline 9.660904 & -72.008445 & 111.4 & $11.029 \pm 0.036$ & $385 \pm 8$ & $0.25 \pm 0.31$ & 1923 & 1101113 & & \\
\hline 9.794922 & -71.569425 & 42.1 & $14.554 \pm 0.017$ & & & 1923 & 1101113 & & Long term drop \\
\hline 9.828878 & -70.131865 & 356.8 & $11.624 \pm 0.384$ & $320 \pm 4$ & $0.51 \pm 0.38$ & 1720 & 1101113 & & \\
\hline 9.887294 & -70.295891 & 428.8 & $11.502 \pm 0.132$ & $386 \pm 6$ & $0.35 \pm 0.21$ & 1721 & 1101113 & & \\
\hline 10.067795 & -73.020987 & 291.6 & $11.568 \pm 0.056$ & $482 \pm 7$ & $0.43 \pm 0.23$ & 1619 & 11011 & 489 & \\
\hline 10.137255 & -73.477785 & 100.0 & $11.423 \pm 0.032$ & $566 \pm 5$ & $0.34 \pm 0.04$ & 1622 & 110111213 & 566 & \\
\hline 10.147153 & -73.324740 & 75.1 & $12.085 \pm 0.028$ & $507 \pm 3$ & $0.55 \pm 0.07$ & 1621 & 1101113 & 515 & \\
\hline
\end{tabular}

Notes. Column 1 lists the coordinates (right ascension and declination) of the source in decimal degrees. Column 2 lists the reduced $\chi^{2}$ statistics. Column 3 lists the mean $K$-band magnitude. Column 4 lists the pulsation period with error. Column 5 lists the amplitude in the $K$-band. Column 6 lists the number of available data points. The first number indicates the number from the VMC survey, the second the total number. Column 7 lists the references for the available data points. Column 8 gives the initial period used in the LC fitting (overwriting any period found from the Fourier analysis). This value comes from the literature, see Table A.2. Column 9 lists any remarks on the source or the LC fitting. Sometimes possible alternative periods are given ("Palt"), or the long secondary period ("Plsp"). The full table is available at the CDS.

References. (1) Present work from the VMC survey; (10) 2MASS (Cutri et al. 2003); (11) 2MASS6X (Cutri et al. 2012); (12) IRSF (Kato et al. 2007); (13) DENIS Consortium (2005); (31) Zijlstra et al. (1996); (32) van Loon et al. (1997); (33) Reid (1991); (34) Wood et al. (1992); (35) Whitelock et al. (2003); (36) Whitelock et al. (1989); (37) Peter Wood and Greg Sloan (priv. comm.); (38) Wood et al. (1983); (39) Hughes \& Wood (1990); (40) Reid et al. (1995); (41) Reid et al. (1990); (42) Glass \& Reid (1985); (43) Nishida et al. (2000); (44) Frogel et al. (1990); (45) Kamath et al. (2010); (46) Groenewegen \& Blommaert (1998); (47) Tanabé et al. (1997); (48) Ferraro et al. (1995). 

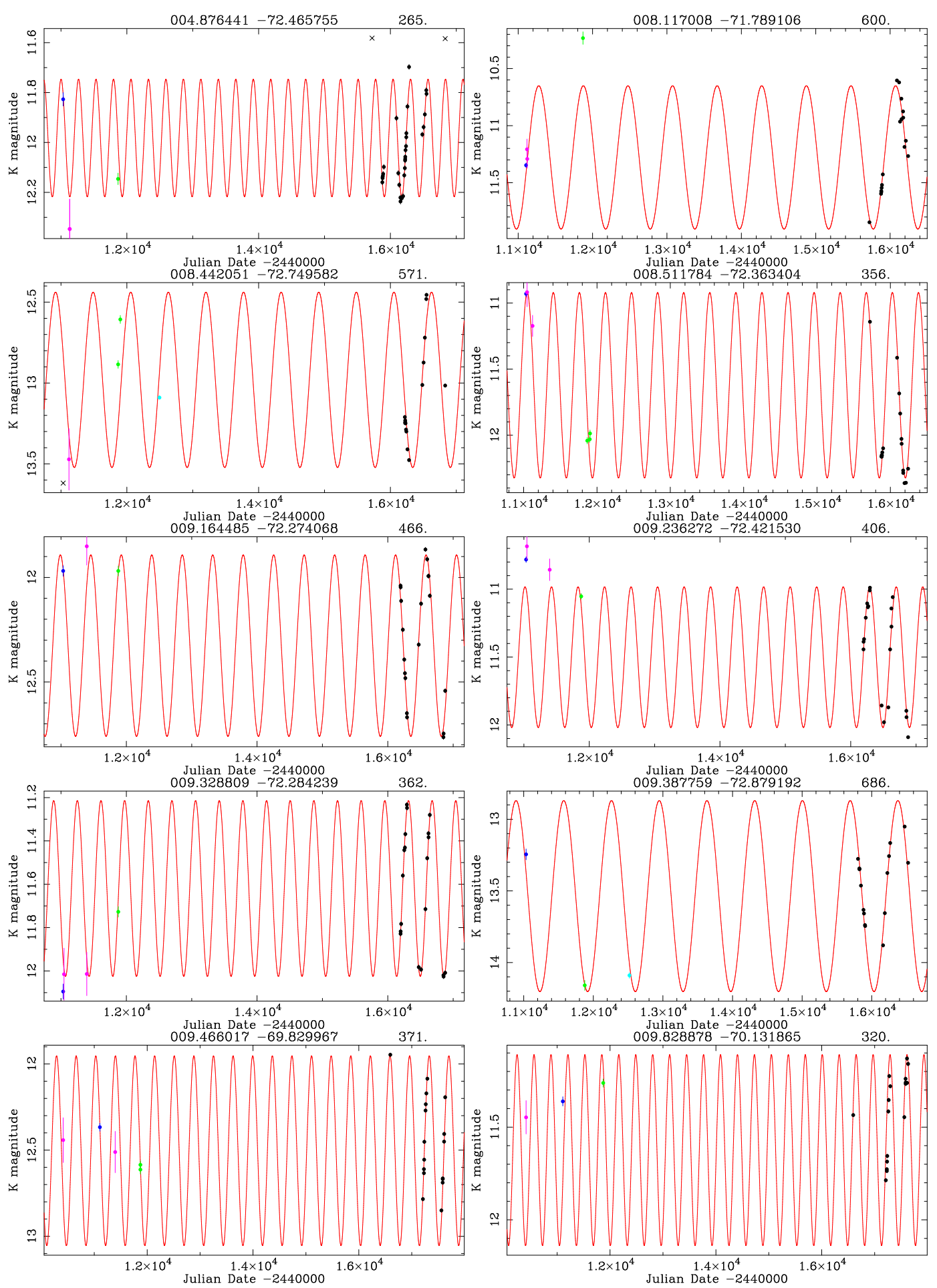

Fig. A.1. Examples of the LC fitting (red solid lines) to the observed time photometry At the top of the plot the identifier and the period (in days) are listed. Data points (with error bars) are identified as black for the VMC, green for 2MASS, dark blue for 2MASS-6X, light blue for IRSF, magenta for DENIS, and orange for other sources (see references in the footnote to Table A.3). Black crosses indicate points that were excluded from the fits. The complete set of LC is available at https://zenodo.org/record/3714889. 


\section{Appendix B: Effect of mixing different $K$-band filters in the analysis}

No attempt was made to bring the various $K$-band measurements into a common system. Various reasons (see Sect. 2.4.2) would make a colour transformation particularly complicated and uncertain in many cases. This is a limitation, and we try to estimate the possible effect now.

Examination of the bolometric corrections computed by Aringer et al. (2009, 2019), limited to the coolest of their spectra $\left(T_{\text {eff }}=2600 \mathrm{~K}\right)$, reveals that the expected magnitude differences between a $K$ passband (from Bessell \& Brett 1989) and a 2MASS $K_{s}$ filter are typically smaller than 0.04 mag for O-rich stars, and smaller than 0.015 mag for C-rich stars. These differences are smaller for stars with hotter photospheres.

We also investigated the synthetic photometry of the best fits to the SEDs and IRS spectra of almost 400 AGB and RSG stars (GS18) that span a very wide range in colour. We compared $K$-band magnitudes in the VISTA, DENIS, IRSF, 2MASS and SAAO systems. For stars with moderate circumstellar reddening $((J-K) \lesssim 2)$ the differences between VISTA and DENIS, and VISTA and 2MASS are of the order quoted above, that is, $\lesssim 0.02 \mathrm{mag}$. For redder stars, the differences are about 0.03-0.04 mag at $(J-K)=5$. The effect in the IRSF filter is weaker, and in the SAAO system it is greater, up to 0.2 mag for the redder stars. These colours are much redder than the transformation formula given in Koen et al. (2007), which are derived in the range $-0.087 \leq(J-K) \leq 1.390$.

The effect of a 0.2 mag change in the externally available photometry (independent of the filter system) was tested on eight stars monitored by Whitelock et al. (2003) without detection in the $J$ band, which based on their $(H-K)$ colour, have an estimated $(J-K) \gtrsim 4$ mag. Their periods range from 550 to 1375 days and their amplitudes from 0.4 to 0.9 mag. The corresponding offset was applied to each of the stars considered, and the period analysis repeated. The absolute differences in the mean magnitudes are 0.01-0.07 mag, and this corresponds to at most a $1.2 \sigma$ difference with respect to the quoted error bar (for six stars the difference is $<0.5 \sigma)$. The absolute differences in periods are $1-8$ days, and this corresponds to at most a $1.8 \sigma$ difference with respect to the quoted error bar (for six stars the difference is $<0.3 \sigma$ ). The absolute differences in amplitudes are $0.01-0.06 \mathrm{mag}$, and this corresponds to at most a $1.3 \sigma$ difference with respect to the quoted error bar (for five stars the difference is $<0.3 \sigma$ ).

We threfore conclude that the differences between different filter systems is in most cases smaller than the quoted error bars and do not influence the results of this paper.

\section{Appendix C: Spectral energy distributions and template fitting}

To obtain insights into the nature of the objects, the SEDs were constructed and compared in a quantitative way to the synthetic SEDs of sources of known composition. For the AGB stars we used as templates the synthetic photometry of the best fits to the SEDs and Spitzer spectra of O-rich AGB stars, RSGs, and C-rich AGB stars from GS18, which are 82, 76, and 204 objects, respectively. These stars cover a wide range in effective temperatures, MLRs and dust compositions. The distinction made in GS18 between O-rich AGB stars and RSGs is based on a number of properties, including luminosity and pulsation period, following the discussion in Sect. 5.1 in Groenewegen et al. (2009).

From Table A.1 and the discussion in Sect. 4.1 it is clear that the main interlopers among possible LPVs on the AGB are
YSOs, but objects classified as HII regions, hot stars (Be stars or blue supergiants (BSG)), Wolf-Rayet (WR) stars, or postAGB/PN stars might also be among the sample of 254 candidate LPVs. To represent the types of sources in the templates, a few sources were picked and analysed in detail, as outlined in the next section.

\section{C.1. Fitting of some non-AGB templates}

One PN, one Wolf-Rayet star, one H II region, three blue BSGs, and six (candidate) YSOs with IRS spectra were selected for a detailed study from the objects in Table A.1. The exception was iras05216 (80.37_-67.85), which was initially considered by GS18 as an AGB star but was eliminated in the process because its SED and IRS spectrum were not consistent with that of an AGB star. Information on these stars is provided in Table C.1.

We followed GS18 and fitted the SEDs and the Spitzer IRS spectra with the code more of DUSTY (MoD, Groenewegen 2012), an extension of the radiative transfer code DUSTY (Ivezić et al. 1999). For a given set of photometry and spectra as input data the programme determines the best-fitting luminosity, dust optical depth, dust temperature at the inner radius, and slope of the density profile.

The stars are hotter than AGB stars and the PHOENIX model atmospheres (Hauschildt et al. 1999) were used to represent the central $\operatorname{star}^{8}$. The dust is a combination of amorphous silicates, corundum, and metallic iron, or amorphous carbon and silicon carbide similar to that in GS18. The proportions between the species were chosen so that they fit the dust continuum and broad dust features of the Spitzer spectrum reasonably well. The actual dust composition may be more complicated, but it is not our aim to study this in detail here.

The fits are shown in Fig. C.1. Overall, the fits are reasonable representations of the photometric data, and this is the main purpose of this exercise. The synthetic photometry corresponding to these fits can serve as representative templates.

There are also clear shortcomings. For example, the spectra show polycyclic aromatic hydrocarbon and other features that are not included in the model. In addition, DUSTY and MoD are one-dimensional codes, but the geometry around the YSOs is often clearly non-spherical. For this reason the fit parameters are not discussed in detail because they are sometimes unphysical, such as dust temperatures at the inner radius above $2000 \mathrm{~K}$.

\section{C.2. Fitting the SEDs to the templates}

Given a template with luminosity $\left(L_{\mathrm{t}}\right)$, distance $\left(d_{\mathrm{t}}\right)$ and synthetic absolute magnitudes in many filters $\left(M_{\mathrm{t}}\right)$ and an object with observed absolute magnitudes with errors $\left(M_{\mathrm{o}}, \sigma\right)$ for an assumed distance $\left(d_{0}\right)$, the comparison between template and object is

$\chi^{2}=\sum\left(\left(M_{\mathrm{t}}+o-M_{\mathrm{o}}\right) / \sigma\right)^{2}$,

where the sum runs over the observed magnitudes in different filters. Because the template was originally fitted to the photometric and spectroscopic data of a star for a certain distance resulting in a best-fitting luminosity there is a degeneracy as a template could fit any observed SED by a simple offset $(o)$ in magnitude.

Setting the derivative of $\chi^{2}$ to zero, the offset that will provide the best fit is

$o=\sum\left(m_{\mathrm{t}}-m_{\mathrm{o}}\right) / \sigma^{2}$,

\footnotetext{
8 https://phoenix.ens-lyon.fr/Grids/BT-NextGen/ SPECTRA/
} 
Table C.1. SED and spectra fitted to some non-AGB stars.

\begin{tabular}{llllll}
\hline \hline $\begin{array}{l}\text { RA } \\
(\mathrm{deg})\end{array}$ & $\begin{array}{c}\text { Dec } \\
(\mathrm{deg})\end{array}$ & Name & $\begin{array}{l}\text { SIMBAD } \\
\text { Object type }\end{array}$ & $\begin{array}{l}\text { IRS } \\
\text { Classification }\end{array}$ & Reference \\
\hline 72.90 & -67.08 & SMP LMC 11 & PN & CPAGB (4) & $\begin{array}{l}\text { Sdopted } \\
\text { classification }\end{array}$ \\
81.51 & -67.49 & HD 36402 & WR & WR (4) & Westerlund \& Smith (1964) \\
81.05 & -68.49 & IRAS 05244-6832 & YSO & HII (4), HII (7) & Kastner et al. (2008) \\
13.53 & -72.69 & LHA 115-S 18 & Em & Be (2), B[e] star (3) & Zickgraf et al. (1989) \\
74.40 & -67.79 & LHA 120-S 12 & BSG & B[e] (4) & Zickgraf et al. (1986) \\
74.19 & -69.84 & HD 268835 & BSG & B[e] (4) & Zickgraf et al. (1986) \\
78.70 & -67.20 & IRAS 05148-6715 & YSO & YSO1 (4), YSO1 (7) & Oliveira et al. (2009) \\
83.16 & -69.51 & [RP2006] 774 & YSO & YSO4 (4), YSO4 (7) & Gruendl \& Chu (2009), Reid (2014) \\
84.00 & -67.75 & 2MASS J05360241-6745171 & YSO candidate & YSO4 (4), YSO4 (7) & Reid (2014) \\
85.14 & -69.41 & 2MASS J05403400-6925099 & YSO & YSO4 (4), O Group (5) & Seale et al. (2009) \\
73.19 & -69.19 & IRAS 04530-6916 & YSO candidate & B[e] (4), F Group (5) & Seale et al. (2009) \\
80.37 & -67.85 & IRAS 05216-6753 & PN & H II (4) & Whitney et al. (2008) \\
\hline
\end{tabular}

Notes. Column 1 gives the right ascension and declination. Column 2 gives the name of the object. Column 3 gives the object type listed in SIMBAD. Column 4 gives the classification based on the IRS spectrum, copied from Table A.1. Column 5 gives a reference supporting the adopted classification listed in Col. 6.

References. (2) Kraemer et al. (2017); (3) Ruffle et al. (2015); (4) Jones et al. (2017); (5) Seale et al. (2009); (7) Woods et al. (2011).

which is the weighted mean difference between observed and template magnitudes.

The SEDs of the 254 targets were compared in this way to a total of 374 templates. As additional constraint, we imposed that in order to be a valid template a $\mathrm{C}$ star had to have a predicted luminosity above $1000 L_{\odot}$ and below $70000 L_{\odot}$, an O-rich AGB star had to have a luminosity higher than $500 L_{\odot}$, and for an O-rich RSG, it had to be above $5000 L_{\odot}$. This resulted in an ordered list of $\chi^{2} \mathrm{~s}$ with the type of the template and the luminosity of the target (for an assumed distance of $50 \mathrm{kpc}$ to the LMC and $61 \mathrm{kpc}$ for the objects in the SMC).

Fifty objects of the 254 were fitted by GS18. This allowed us to check the level of contamination: if a star is a known $\mathrm{C}$-rich object, is the best-fitting template that of a $\mathrm{C}$ star, and at what level of $\chi^{2}$ is a non-C star considered a possible match. This is not a trivial exercise because the SEDs used in GS18 and constructed here are not the same. For example, GDR2 photometry and VMC magnitudes were not used by GS18. Instead NIR photometry was taken from the available 2MASS, 2MASS6X, IRSF, and DENIS data. In addition, the fits in GS18 were made to the photometry and Spitzer spectra, which leads to different synthetic magnitudes than if only the photometry were fitted, as we did here. For the 35 known $\mathrm{C}$ stars, the best-fitting template is always that of a C star and the best-fitting O-rich template has a $\chi^{2}$ that is (when ordered) 1.36, 1.99, 2.05, etc. times larger than the $\chi^{2}$ of the best-matching template. Of the 15 known O-rich stars, the best-fitting template is always that of an O-rich star, and the bestfitting C-rich template has a $\chi^{2}$ that is $1.28,1.31,1.32,1.51,1.65$, etc. times larger than the $\chi^{2}$ of the best-matching template.

Based on this result, the list of templates with a $\chi^{2}$ lower than 1.5 times that of the best-fitting template was retained and used in the classification. In this case, 34 stars were classified as "C" (all templates are of C stars), one as "C (AGB)" (the best-fitting template is C, and the alternative(s) is (are) (an) O-rich AGB stars or RSGs), 12 as "O" (all templates are O-rich AGB stars or RSGs), and 3 as "O (AGB)" (the best-fitting template is that of an O-rich AGB star or RSG, and the alternative(s) is (are) C stars).

Gullieuszik et al. (2012) also reported that the classification of AGB stars based on a fit to the SED gives reliable results, and that the success rate of classification is better for $\mathrm{C}$ than for O stars. They fitted the SEDs of 374 stars in one VMC field selected to be AGB stars based on CCDs and CMDs both with a $\mathrm{C}$ star atmosphere model and carbonaceous dust grains, and an
O star model and silicate dust grains. They then selected the best model based on a $\chi^{2}$ comparison. Eighty-seven stars in the field were spectroscopically identified as C stars and the SED fitting classified $87 \%$ of them correctly, even $100 \%$ for stars with $(J-$ $\left.K_{s}\right)>1.5 \mathrm{mag}$. They also fitted the SEDs of stars with a known classification from Spitzer spectra in the MCs. The classification was correct in more than $90 \%$ of the cases for $\mathrm{C}$ stars and about $75 \%$ for the O-rich stars.

When the list of templates includes a non-AGB star, this is marked by OTHER, for example, "C(OTH)". The best-fitting template could be a YSO, and in this case, the classification might be "YSO" (all templates are those of YSOs), "YSO(AGB)" (the best-fitting template is that of a YSO, and the alternatives are only O-rich AGB stars, RSGs, or C-rich stars), or "YSO(OTH)" (the best-fitting template is that of a YSO, and the alternatives could be (an) AGB star(s), RSG(s), WR, Be star, HII, or PN).

The results of the template fitting are listed in Col. 10 in Table C.2 with the predicted luminosity in Col. 11 (the error in $L$ is estimated to be $15 \%$ based on the typical scatter in luminosity among the best-fitting templates). The SEDs of all 254 objects with their best-matching templates are plotted in Fig. C.2. In total, 197 stars are classified as C-rich (in two cases the matching templates include O-rich stars, and in three cases the matching templates include "other"; objects). The luminosities are between 1000 and $29000 L_{\odot}$, in agreement with GS18, who derived luminosities for $225 \mathrm{C}$ stars between 1125 and $56700 L_{\odot}$. All three objects marked as " $\mathrm{C}(\mathrm{OTH})$ " are at relatively low luminosities between 1250 and $2850 L_{\odot}$, and based on a visual inspection of their SED and additional information (one object is classified as an emission-line object) the three are classified as likely non-AGB stars. In total, 22 stars are classified as O-rich (in five cases the matching templates include C-rich stars, and in two cases the matching templates include "other" objects). The luminosities are between 7000 and $98000 L_{\odot}$, with one exception $\left(1500 L_{\odot}\right)$, in agreement with GS18. For 82 stars classified as Orich AGB stars, they found a range between 1500 and $107000 L_{\odot}$, and for 76 stars they classified as RSG, a range from 24500 to $\sim 250000 L_{\odot}$. This means that 217 stars are very likely AGB stars, and the period analysis has revealed LPV like properties.

Twenty stars are classified as YSOs; in five cases AGB stars are among the best-fitting templates. Fifteen have luminosities between 400 and $2800 L_{\odot}$, while five have luminosities between 4500 and $20000 L_{\odot}$. Two stars are classified as PNe, six as HII regions and seven as Be stars. 
M. A. T. Groenewegen et al.: Pulsation periods of enshrouded AGB stars in the Magellanic Clouds
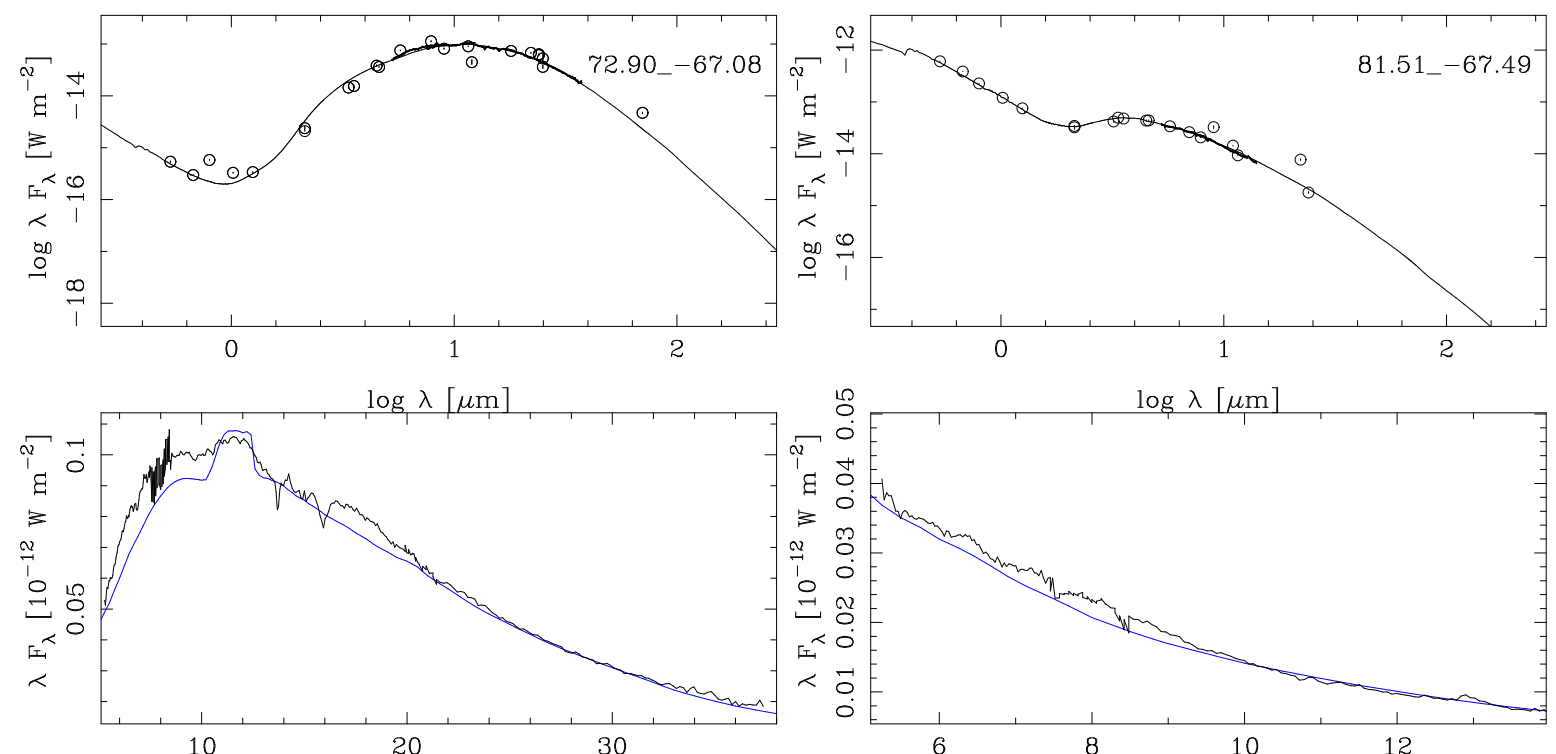

$\lambda[\mu \mathrm{m}]$
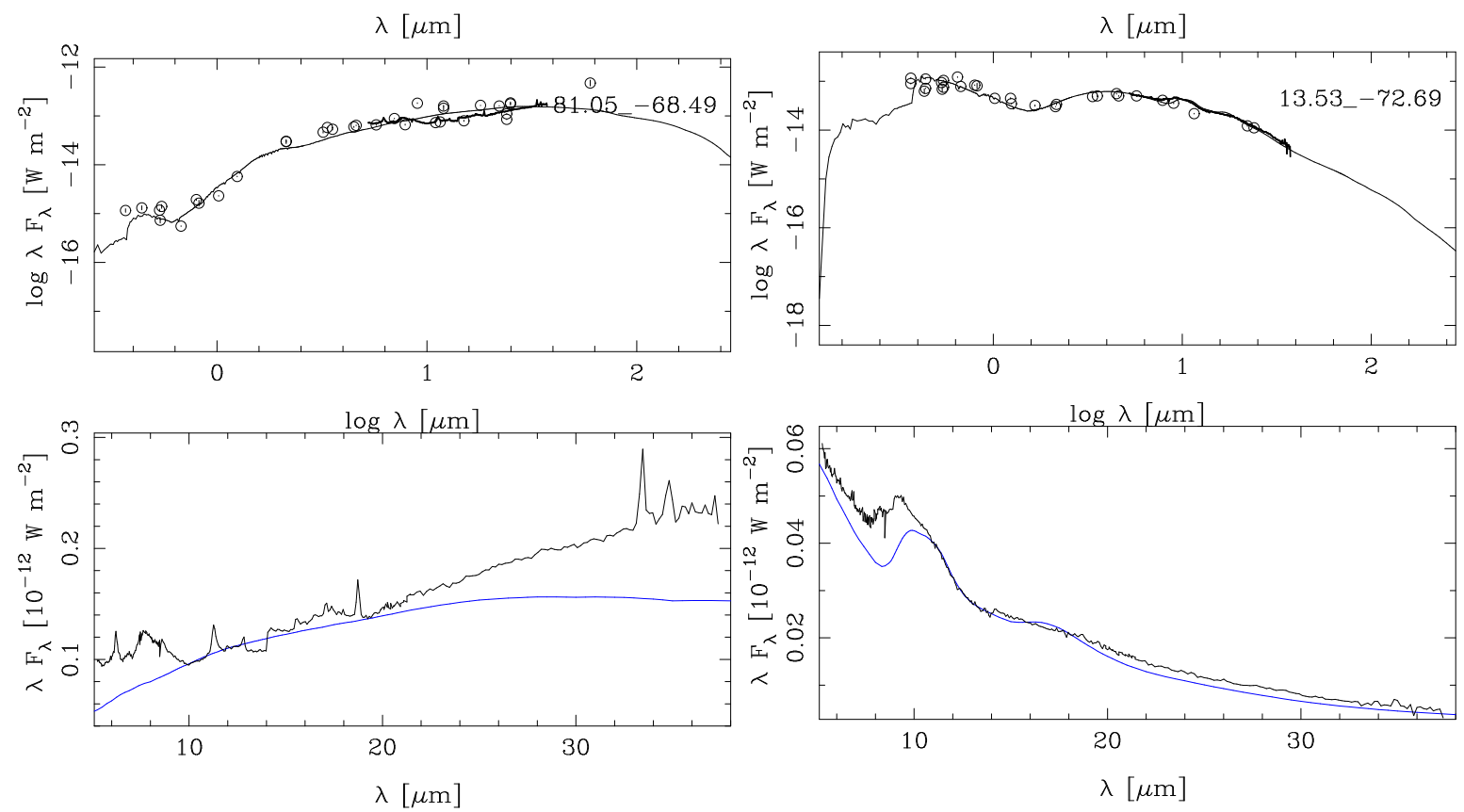

Fig. C.1. SEDs and Spitzer spectra of a C-rich post-AGB/PN star (top left), a WR star (top right), an HII region (bottom left), and a Be star (bottom right). See the notes to Table C.1 for the spectroscopic classification. In the panels with the Spitzer IRS spectra, the models (blue lines) are scaled to the observed spectra based on the average flux in the 12-13 $\mu \mathrm{m}$ region. For simplicity, the identifiers are the RA and Dec from Table A.1 truncated to two decimal figures. 
A\&A 636, A48 (2020)
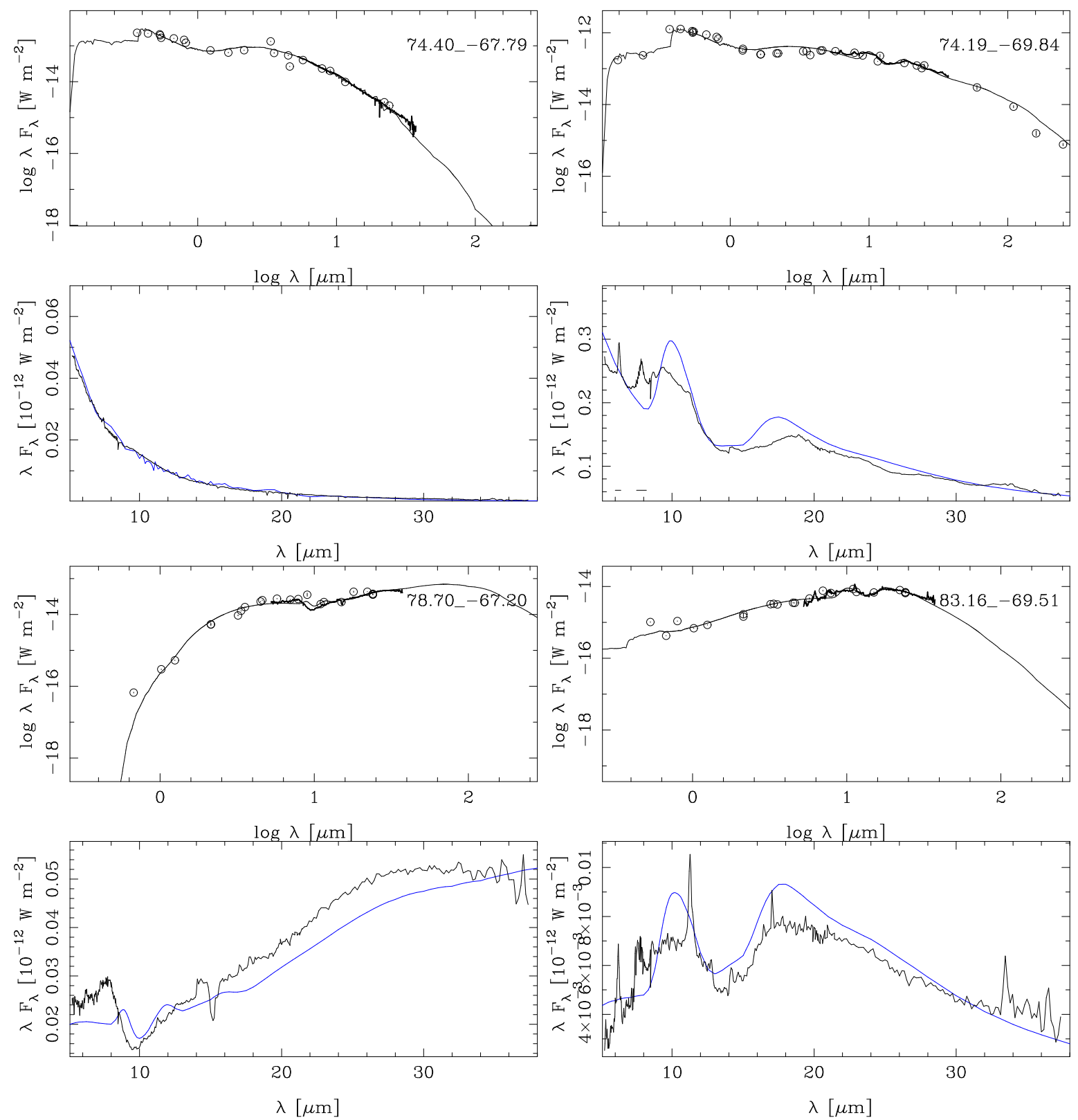

Fig. C.1. continued. The SEDs and Spitzer spectra of two Be stars (top panels) and two YSOs (bottom panels). See the notes to Table C.1 for the spectroscopic classification. 
M. A. T. Groenewegen et al.: Pulsation periods of enshrouded AGB stars in the Magellanic Clouds
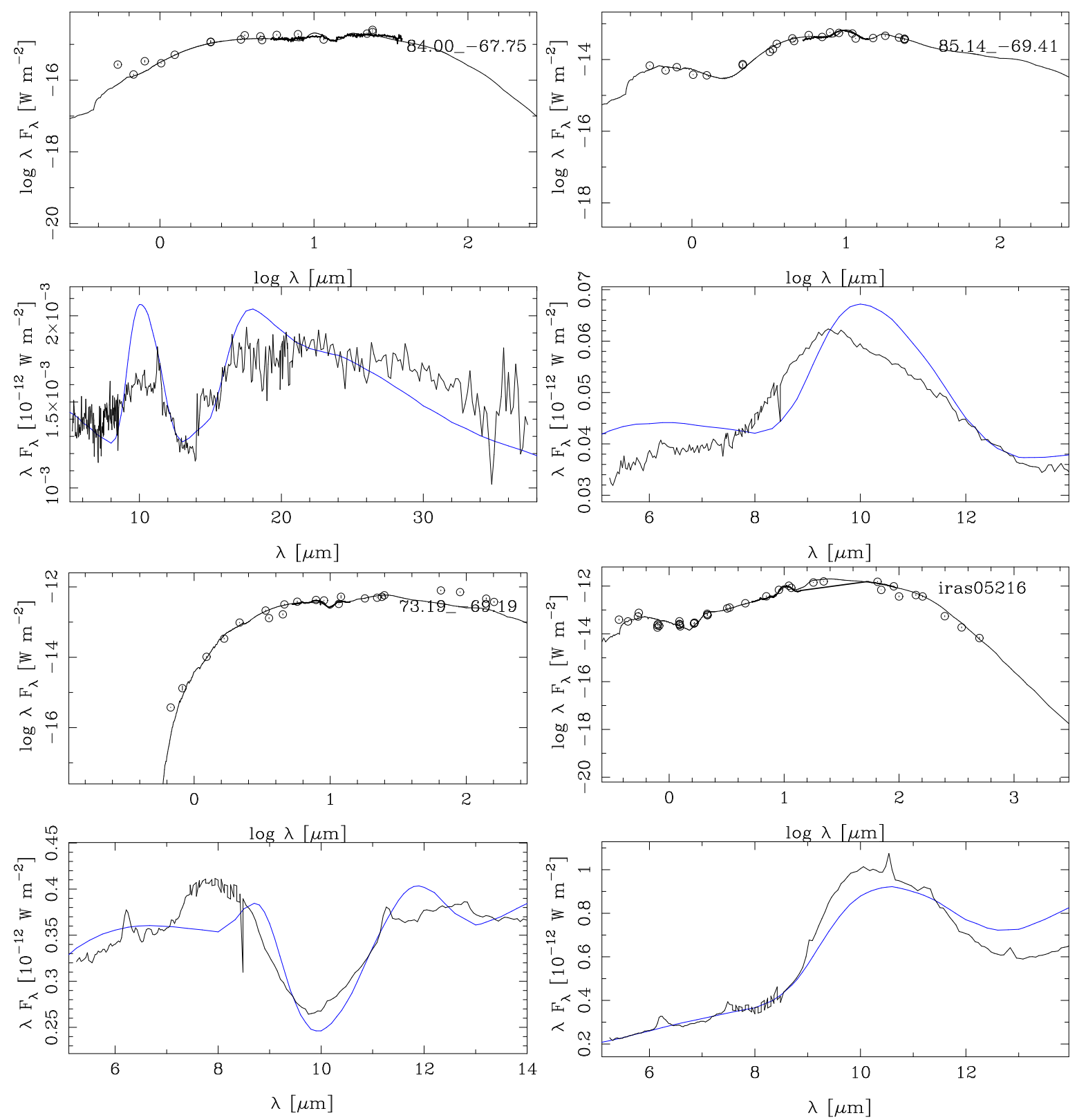

Fig. C.1. continued. The SEDs and Spitzer spectra of four YSOs. See the notes to Table C.1 for the spectroscopic classification. 


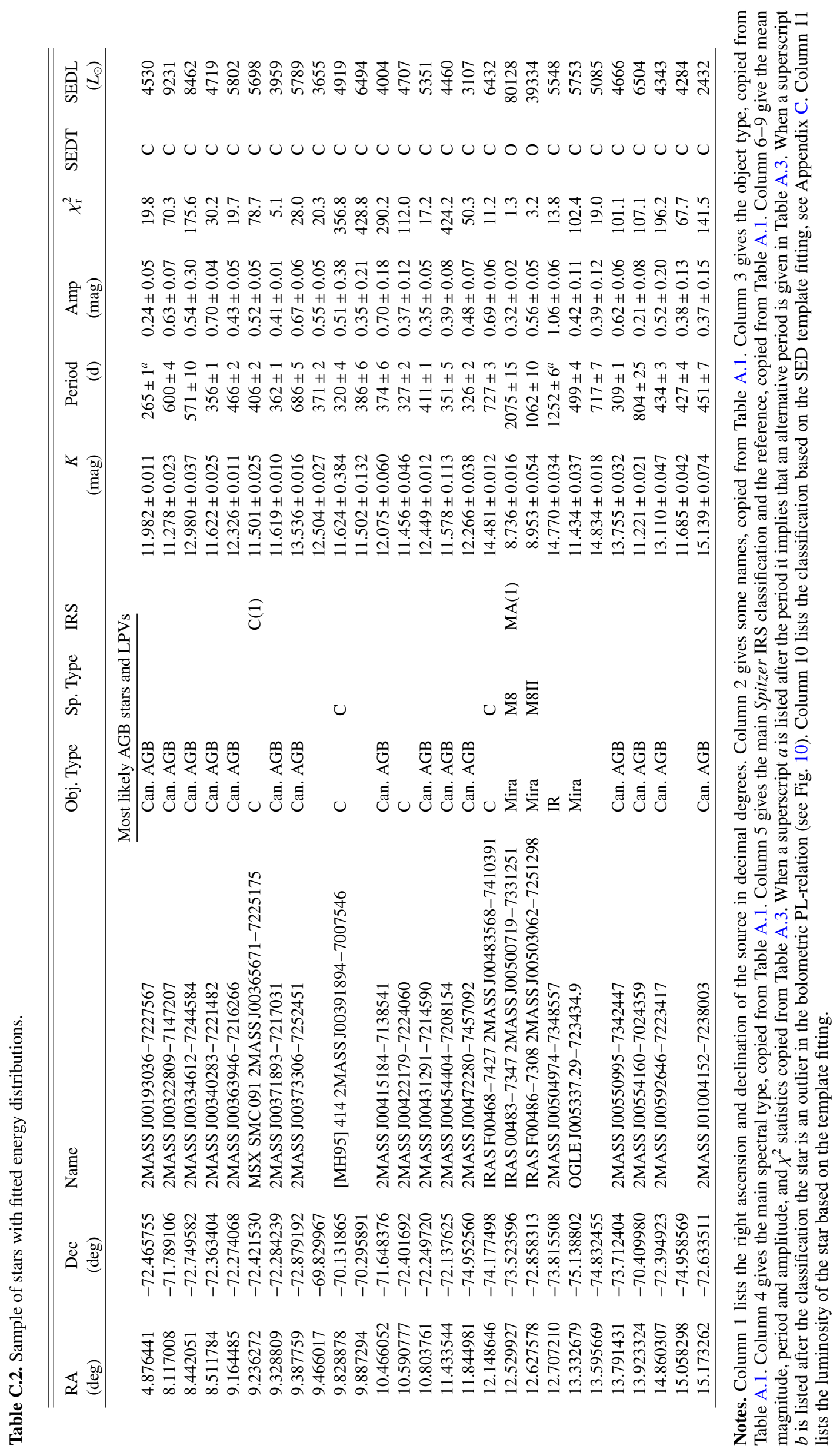


M. A. T. Groenewegen et al.: Pulsation periods of enshrouded AGB stars in the Magellanic Clouds

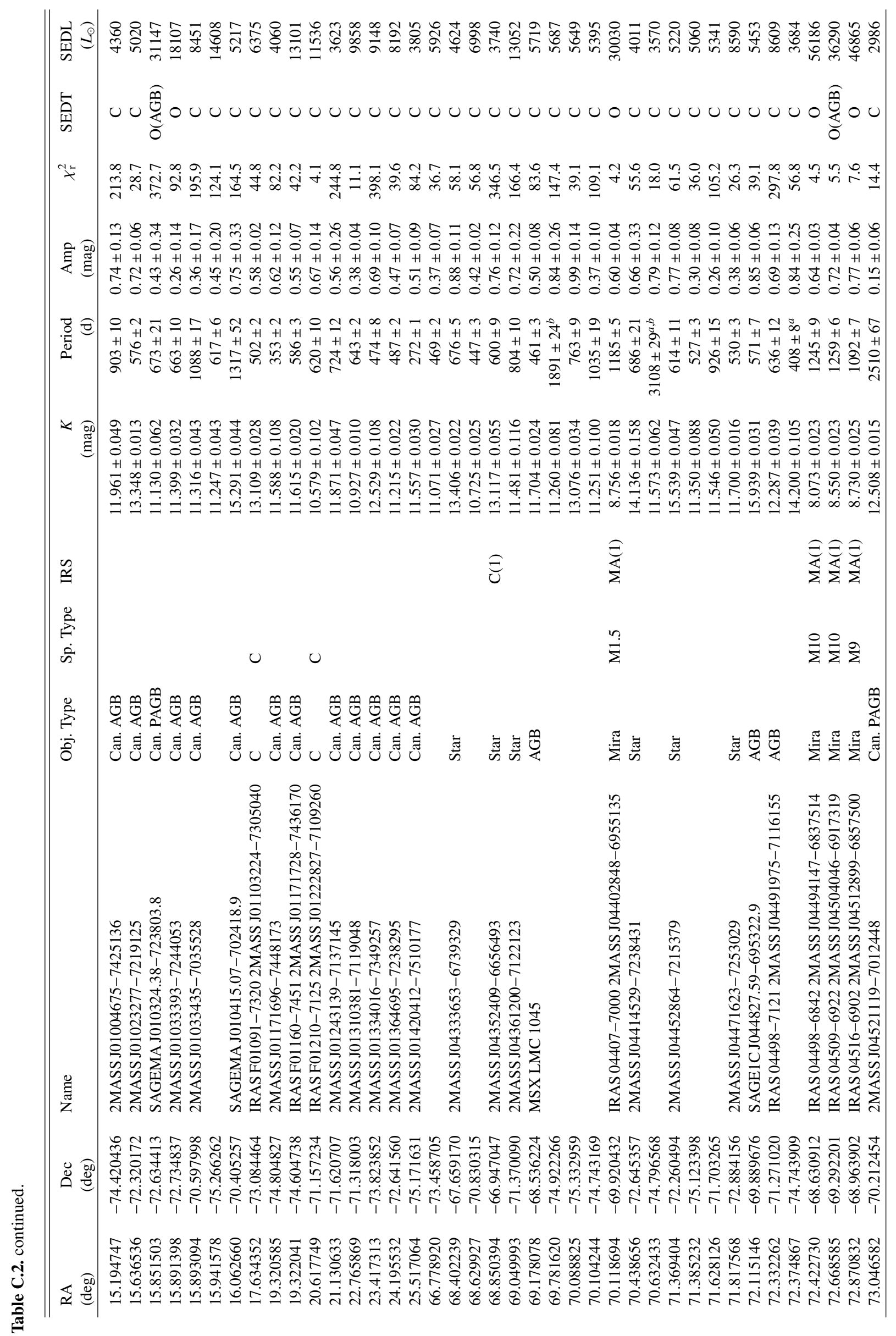




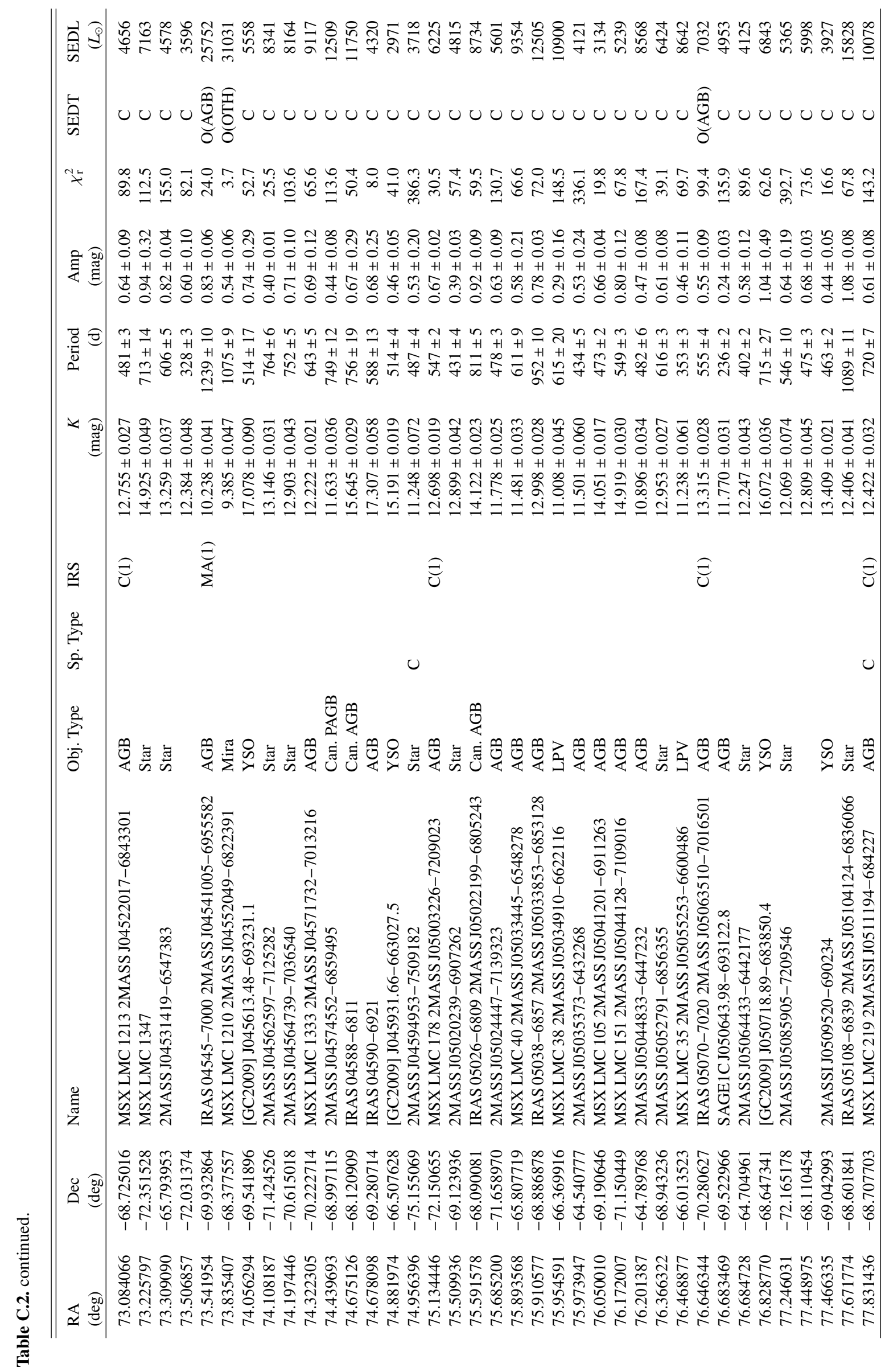




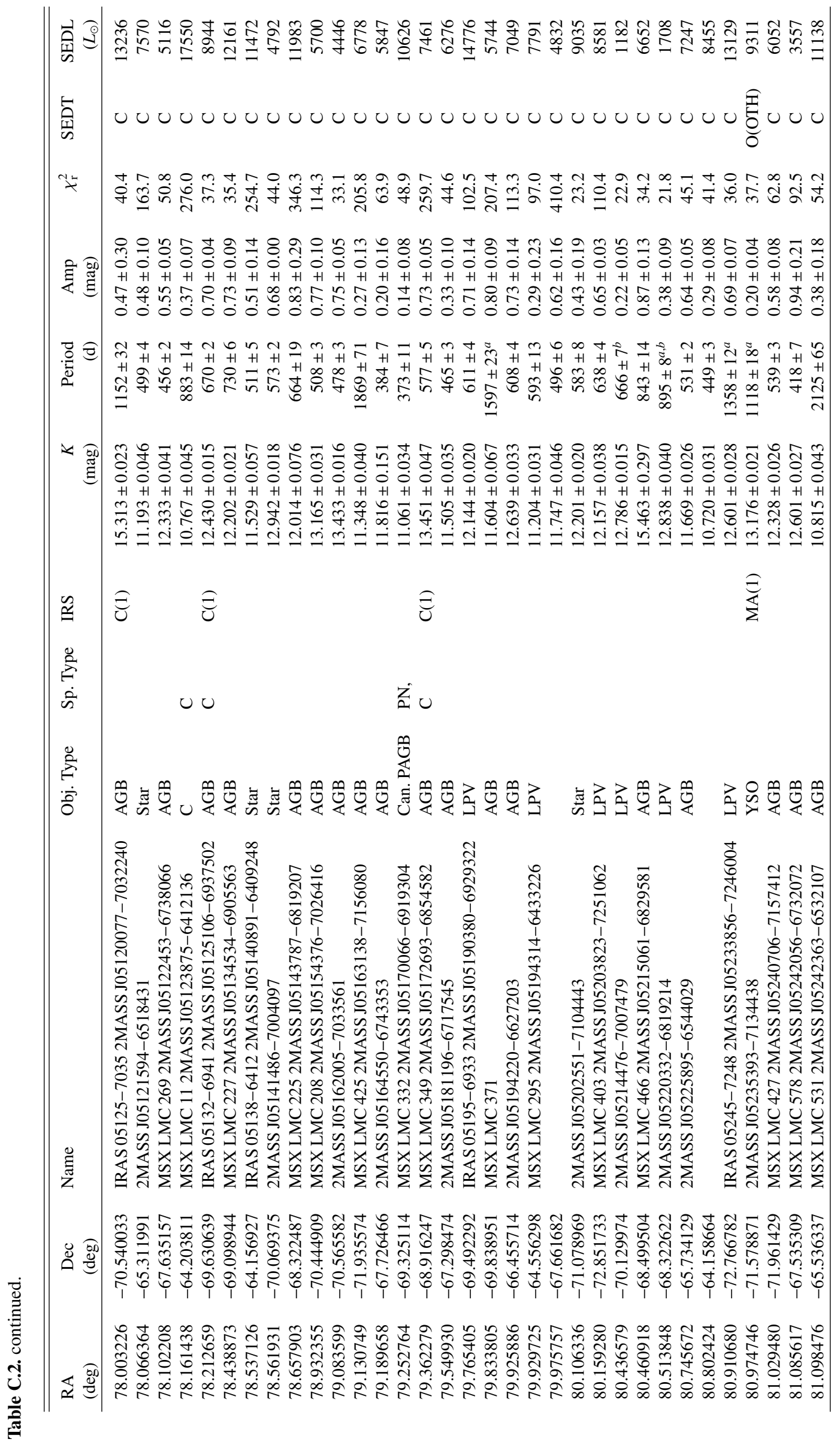




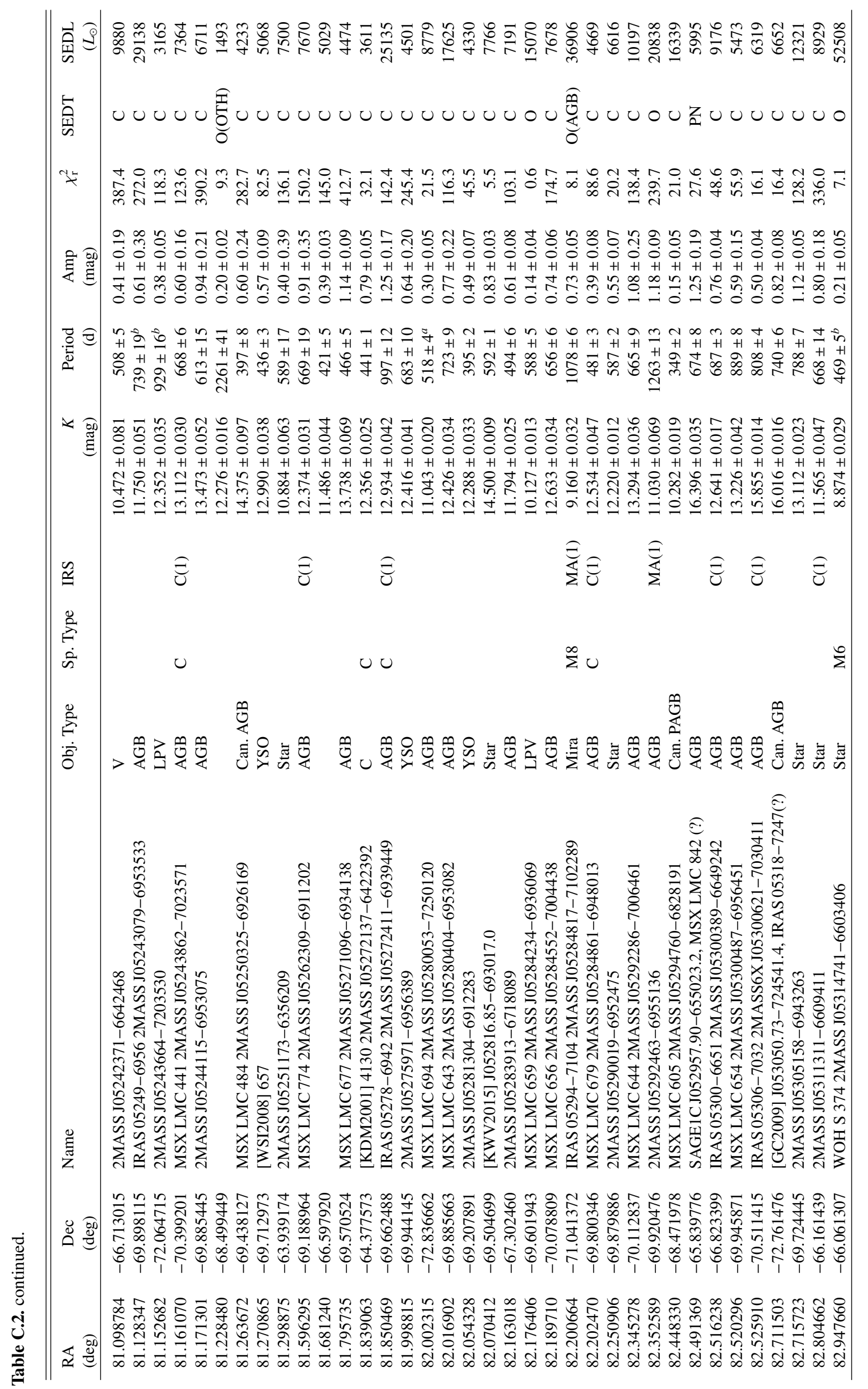


M. A. T. Groenewegen et al.: Pulsation periods of enshrouded AGB stars in the Magellanic Clouds

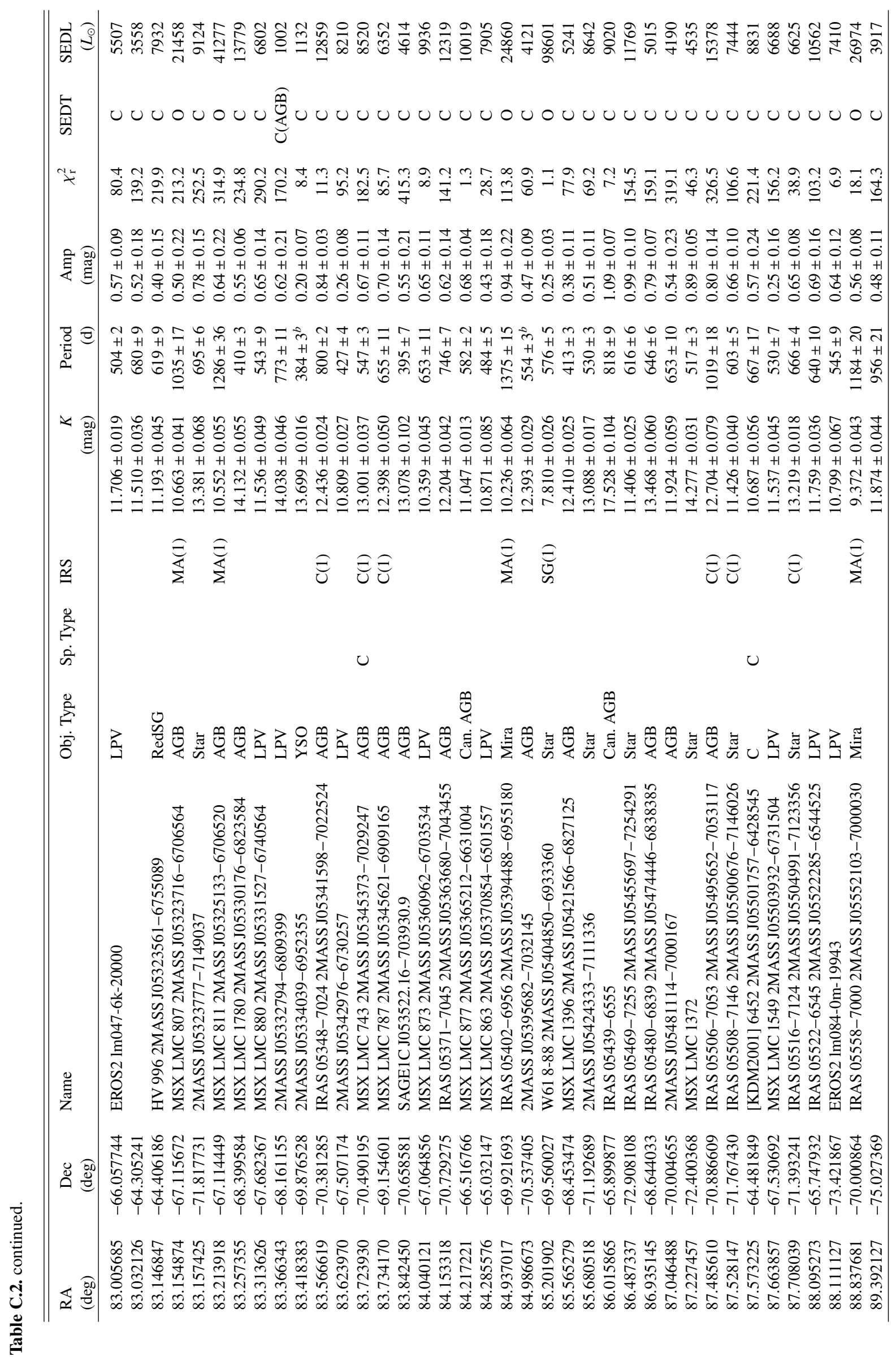




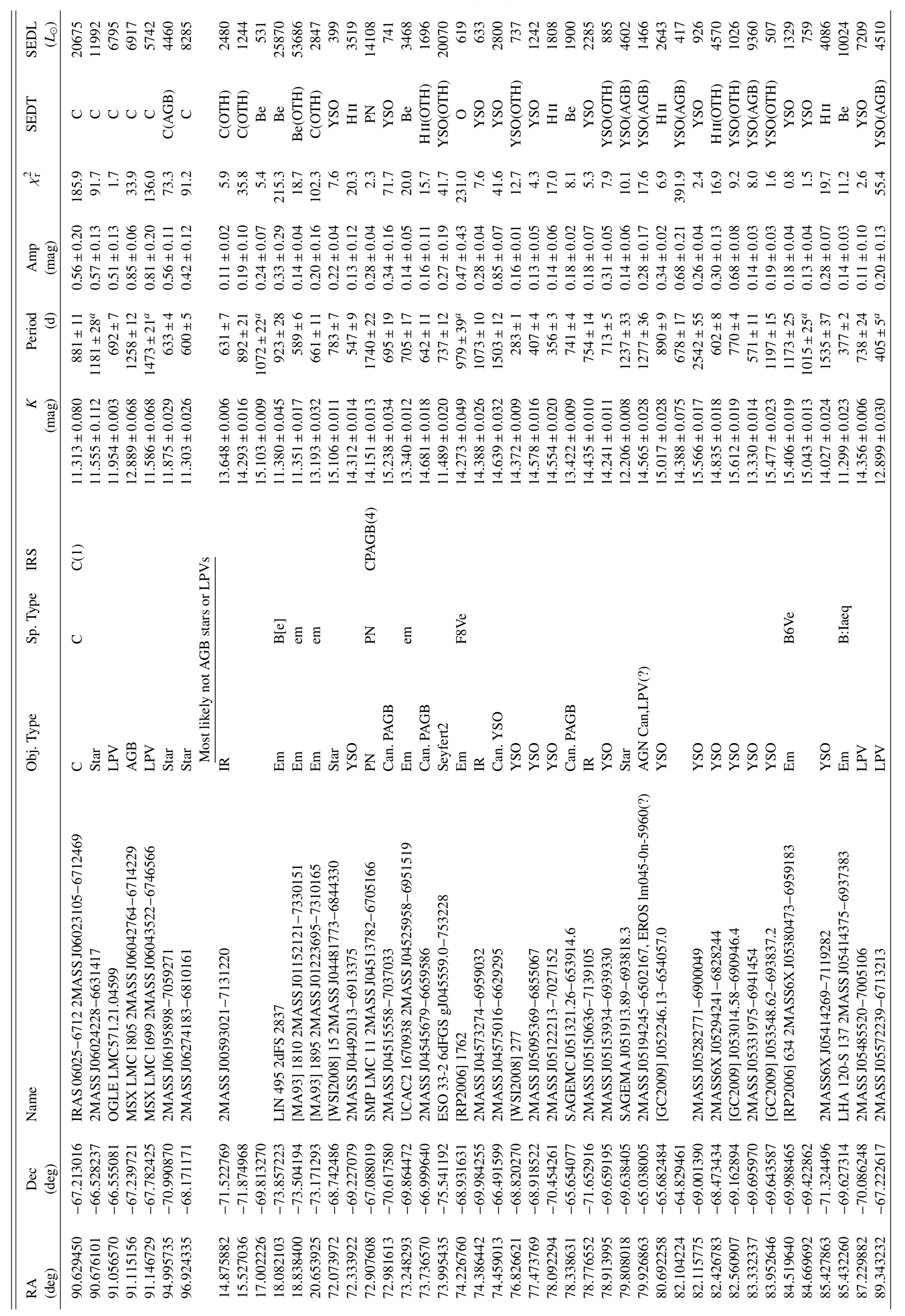


M. A. T. Groenewegen et al.: Pulsation periods of enshrouded AGB stars in the Magellanic Clouds
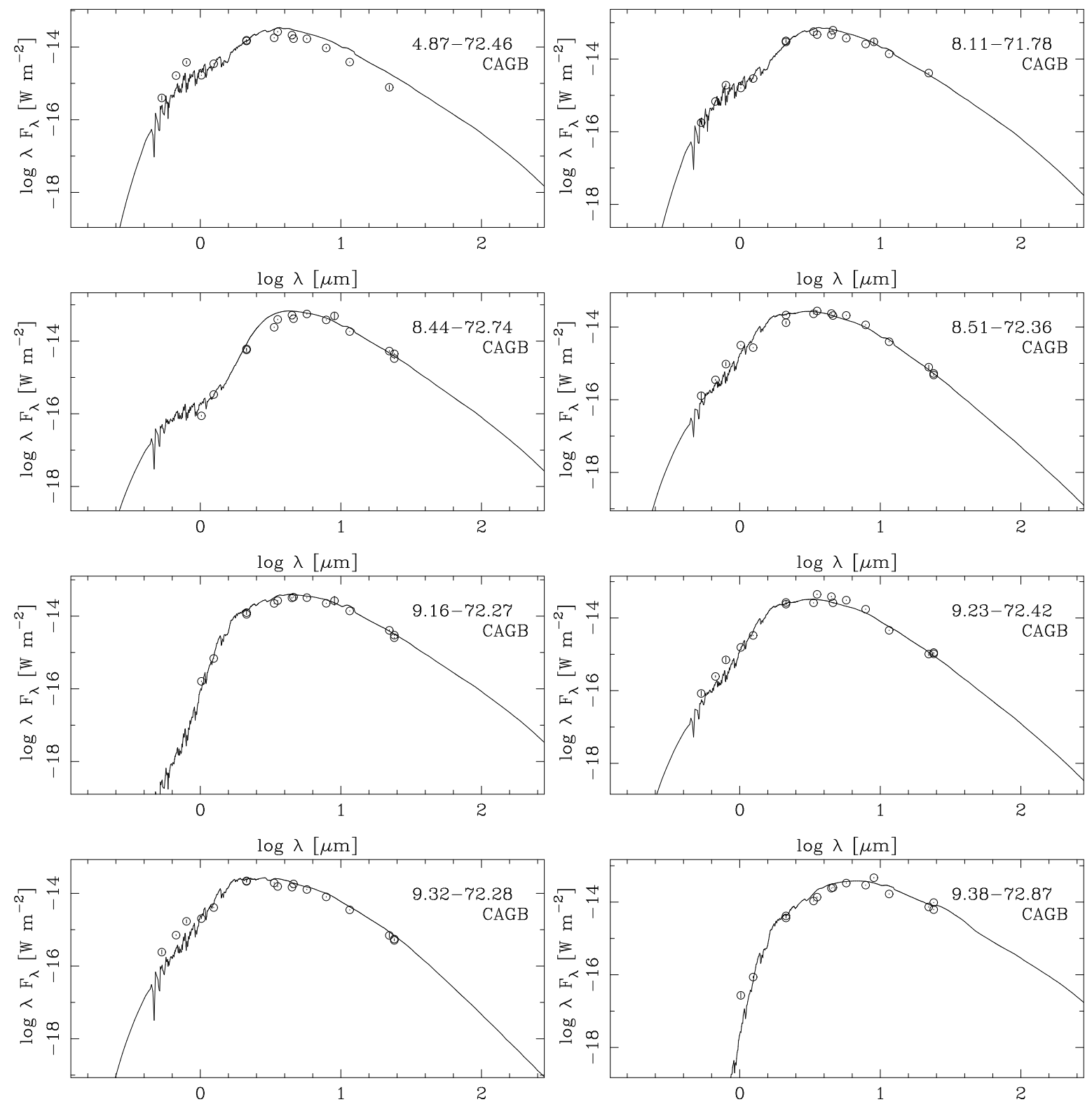

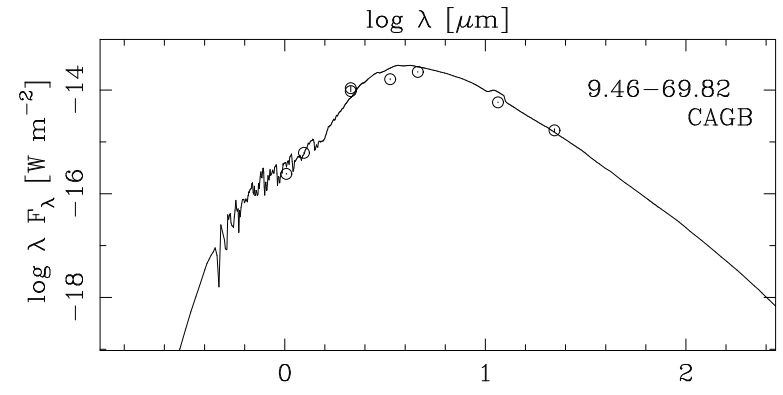

$\log \lambda[\mu \mathrm{m}]$

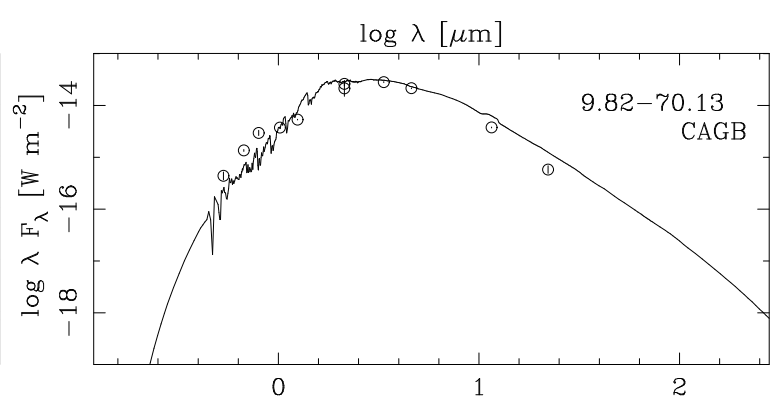

$\log \lambda[\mu \mathrm{m}]$

Fig. C.2. Examples of the fitting of a template (the solid line) to the observed photometry. The top right corner lists the identifier of the star (RA and Dec truncated to two decimal figures), and below it the type of the template. The complete set of 254 SEDs is available at https: //zenodo.org/record/3714889. 


\section{Appendix D: Results of the fits to synthetic spectra from Nanni et al. (2019)}

Table D.1. Results from the SED fitting performed to synthetic spectra from Nanni et al. (2019) for all AGB candidates selected in this work.

\begin{tabular}{|c|c|c|c|c|}
\hline $\begin{array}{l}\text { RA } \\
(\mathrm{deg})\end{array}$ & $\begin{array}{c}\text { Dec } \\
(\mathrm{deg})\end{array}$ & Name & $\begin{array}{l}\dot{M} \\
\left(10^{-6} M_{\odot} \mathrm{yr}^{-1}\right)\end{array}$ & $\begin{array}{l}\text { Luminosity } \\
\left(L_{\odot}\right)\end{array}$ \\
\hline 4.876441 & -72.465755 & 2MASS J00193036-7227567 & $1.76 \pm 0.67$ & 3162 \\
\hline 8.117008 & -71.789106 & 2MASS J00322809-7147207 & $5.0 \pm 1.5$ & 7943 \\
\hline 8.442051 & -72.749582 & 2MASS J00334612-7244584 & 7.94 & 7943 \\
\hline 8.511784 & -72.363404 & 2MASS J00340283-7221482 & 2.51 & 3981 \\
\hline 9.164485 & -72.274068 & 2MASS J00363946-7216266 & 3.16 & 5012 \\
\hline 9.236272 & -72.421530 & MSX SMC 091 2MASS J00365671-7225175 & $3.08 \pm 0.54$ & 5012 \\
\hline 9.328809 & -72.284239 & 2MASS J00371893-7217031 & $1.72 \pm 0.54$ & 3162 \\
\hline 9.387759 & -72.879192 & 2MASS J00373306-7252451 & 11.22 & 5012 \\
\hline 9.466017 & -69.829967 & & $2.57 \pm 0.58$ & 3162 \\
\hline 9.828878 & -70.131865 & [MH95] 414 2MASS J00391894-7007546 & $1.85 \pm 0.33$ & 3981 \\
\hline 9.887294 & -70.295891 & & 1.58 & 5012 \\
\hline 10.466052 & -71.648376 & 2MASS J00415184-7138541 & $2.98 \pm 0.63$ & 3981 \\
\hline 10.590777 & -72.401692 & 2MASS J00422179-7224060 & $1.59 \pm 0.49$ & 3981 \\
\hline 10.803761 & -72.249720 & 2MASS J00431291-7214590 & 2.0 & 3981 \\
\hline 11.433544 & -72.137625 & 2MASS J00454404-7208154 & $1.92 \pm 0.36$ & 3981 \\
\hline 11.844981 & -74.952560 & 2MASS J00472280-7457092 & 1.00 & 2512 \\
\hline 12.148646 & -74.177498 & IRAS F00468-7427 2MASS J00483568-7410391 & $15.8 \pm 4.2$ & 6310 \\
\hline 12.529927 & -73.523596 & IRAS 00483-7347 2MASS J00500719-7331251 & $37.3 \pm 2.6$ & 25120 \\
\hline 12.627578 & -72.858313 & IRAS F00486-7308 2MASS J00503062-7251298 & $4.35 \pm 1.55$ & 25120 \\
\hline 12.707210 & -73.815508 & 2MASS J00504974-7348557 & $14.13 \pm 3.23$ & 5012 \\
\hline 13.332679 & -75.138802 & OGLE J005337.29-723434.9 & $2.12 \pm 0.56$ & 5012 \\
\hline 13.595669 & -74.832455 & & $13.19 \pm 3.48$ & 5012 \\
\hline 13.791431 & -73.712404 & 2MASS J00550995-7342447 & $9.1 \pm 3.2$ & 3981 \\
\hline 13.923324 & -70.409980 & 2MASS J00554160-7024359 & $3.2 \pm 1.1$ & 5012 \\
\hline 14.860307 & -72.394923 & 2MASS J00592646-7223417 & 5.01 & 3981 \\
\hline 15.058298 & -74.958569 & & 1.58 & 3981 \\
\hline 15.173262 & -72.633511 & 2MASS J01004152-7238003 & $2.9 \pm 1.0$ & $2081 \pm 211$ \\
\hline 15.194747 & -74.420436 & 2MASS J01004675-7425136 & $1.85 \pm 0.33$ & 3162 \\
\hline 15.636536 & -72.320172 & 2MASS J01023277-7219125 & $3.56 \pm 1.16$ & 3981 \\
\hline 15.851503 & -72.634413 & SAGEMA J010324.38-723803.8 & $1.36 \pm 0.28$ & $20560 \pm 1220$ \\
\hline 15.891398 & -72.734837 & 2MASS J01033393-7244053 & 1.00 & 14130 \\
\hline 15.893094 & -70.597998 & 2MASS J01033435-7035528 & $3.04 \pm 0.70$ & 6310 \\
\hline 15.941578 & -75.266262 & & $5.34 \pm 0.65$ & 12590 \\
\hline 16.062660 & -70.405257 & SAGEMA J010415.07-702418.9 & $10.7 \pm 1.7$ & 5012 \\
\hline 17.634352 & -73.084464 & IRAS F01091-7320 2MASS J01103224-7305040 & 7.94 & 6310 \\
\hline 19.320585 & -74.804827 & 2MASS J01171696-7448173 & 2.0 & 3162 \\
\hline 19.322041 & -74.604738 & IRAS F01160-7451 2MASS J01171728-7436170 & 11.2 & 11220 \\
\hline 20.617749 & -71.157234 & IRAS F01210-7125 2MASS J01222827-7109260 & $6.7 \pm 2.7$ & 11220 \\
\hline 21.130633 & -71.620707 & 2MASS J01243139-7137145 & 2.51 & 3981 \\
\hline 22.765869 & -71.318003 & 2MASS J01310381-7119048 & $5.11 \pm 0.36$ & 7943 \\
\hline 23.417313 & -73.823852 & 2MASS J01334016-7349257 & 10.0 & 7943 \\
\hline 24.195532 & -72.641560 & 2MASS J01364695-7238295 & 3.16 & 6310 \\
\hline 25.517064 & -75.171631 & 2MASS J01420412-7510177 & 1.26 & 3162 \\
\hline 66.778920 & -73.458705 & & $4.23 \pm 0.98$ & 6310 \\
\hline 68.402239 & -67.659170 & 2MASS J04333653-6739329 & 7.94 & 5012 \\
\hline 68.629927 & -70.830315 & & $3.52 \pm 0.67$ & 6310 \\
\hline 68.850394 & -66.947047 & 2MASS J04352409-6656493 & $3.00 \pm 0.33$ & 3981 \\
\hline 69.049993 & -71.370090 & 2MASS J04361200-7122123 & $10.0 \pm 1.2$ & $13668 \pm 744$ \\
\hline 69.178078 & -68.536224 & MSX LMC 1045 & 2.51 & 5012 \\
\hline 69.781620 & -74.922266 & & 3.16 & 6310 \\
\hline 70.088825 & -75.332959 & & $15.2 \pm 2.5$ & 5012 \\
\hline 70.104244 & -74.743169 & & $3.26 \pm 0.89$ & 5012 \\
\hline 70.118694 & -69.920432 & IRAS 04407-7000 2MASS J04402848-6955135 & $14.4 \pm 3.0$ & 22390 \\
\hline 70.438656 & -72.645357 & 2MASS J04414529-7238431 & $5.1 \pm 1.1$ & 3981 \\
\hline 70.632433 & -74.796568 & & 1.26 & 3162 \\
\hline 71.369404 & -72.260494 & 2MASS J04452864-7215379 & $10.7 \pm 2.8$ & 5012 \\
\hline
\end{tabular}

Notes. Column 1 lists the right ascension and declination of the source in decimal degrees. Column 2 gives some names, copied from Table A.1. Column 3 lists the MLR. Column 4 lists the luminosity. The full table is available at the CDS. 
Table D.1 lists the first entries of the table available at the CDS. It provides the results of the SED fitting performed to synthetic spectra from Nanni et al. (2019) for the 217 objects classified as AGB stars in this work.

The calculation of the uncertainty on the MLR and luminosity is the same as that described by Nanni et al. $(2018,2019)$. The synthetic photometry that best fits the observations provides $\chi^{2}=\chi_{\text {best }}^{2}$. Each of the synthetic fluxes are randomly modified within the observed flux errors, and a new value of $\chi^{2}$ between the observed and modified flux is calculated. Such a procedure is reiterated 100 times and an equal number of $\chi^{2}$ values are computed. From the $100 \chi^{2}$ we extract the minimum and the 34 th values (corresponding to $1 \sigma$ ). The difference between the minimum and the $1 \sigma$ value provides the statistical variation of $\Delta \chi^{2}$ and thus the maximum acceptable value of $\chi^{2}, \chi_{\max }^{2}=$ $\chi_{\text {best }}^{2}+\Delta \chi^{2}$. We then select from the grid of models those that yield a value of $\chi^{2}: \chi^{2} \leq \chi_{\max }^{2}$, and we compute their average and standard deviation, which provides the corresponding uncertainty of the quantity calculated. If fewer than 4 models satisfy the condition $\chi^{2} \leq \chi_{\max }^{2}$, the stellar quantities are assumed to be represented by the best-fitting value and zero uncertainty. 